\title{
TYPE-CHECKING LIVENESS FOR COLLABORATIVE PROCESSES WITH BOUNDED AND UNBOUNDED RECURSION*
}

\author{
SØREN DEBOIS ${ }^{a}$, THOMAS HILDEBRANDT $^{b}$, TIJS SLAATS $^{c}$, AND NOBUKO YOSHIDA $^{d}$ \\ ${ }^{a, b, c}$ IT University of Copenhagen, Rued Langgaards Vej 7, 2300 Copenhagen S, Denmark \\ e-mail address: \{debois@itu.dk,hilde@itu.dk,tslaats@itu.dk\} \\ ${ }^{c}$ Exformatics A/S, Dag Hammerskjölds Allé 13, 2100 København Ø, Denmark \\ ${ }^{d}$ Imperial College London, Department of Computing, 180 Queen's Gate, South Kensington Campus, \\ SW7 2AZ, United Kingdom \\ e-mail address: yoshida@doc.ic.ac.uk
}

\begin{abstract}
We present the first session typing system guaranteeing request-response liveness properties for possibly non-terminating communicating processes. The types augment the branch and select types of the standard binary session types with a set of required responses, indicating that whenever a particular label is selected, a set of other labels, its responses, must eventually also be selected. We prove that these extended types are strictly more expressive than standard session types. We provide a type system for a process calculus similar to a subset of collaborative BPMN processes with internal (databased) and external (event-based) branching, message passing, bounded and unbounded looping. We prove that this type system is sound, i.e., it guarantees request-response liveness for dead-lock free processes. We exemplify the use of the calculus and type system on a concrete example of an infinite state system.
\end{abstract}

2012 ACM CCS: [Theory of computation]: Models of computation; Semantics and reasoningProgram reasoning - Program specifications; Applied computing]: Enterprise computing —Business process management-Business process modeling.

Key words and phrases: Session types, Business processes, Liveness, Bounded recursion, Process algebra, Typing system.

* Full version of Extended Abstract previously presented at FORTE '14.

This work supported in part by the Computational Artifacts project (VELUX 33295, 2014-2017); by the Danish Agency for Science, Technology and Innovation; by EPSRC EP/K011715/1, EPSRC EP/K034413/1, and EPSRC EP/L00058X/1, EU project FP7-612985 UpScale, and EU COST Action IC1201 BETTY.. 


\section{INTRODUCTION}

Session types were originally introduced as typing systems for particular $\pi$-calculi, modelling the interleaved execution of two-party protocols. A well-typed process is guaranteed freedom from race-conditions as well as communication compatibility, usually referred to as session fidelity [21, 34, 32. Session types have subsequently been studied intensely, with much work on applications, typically to programming languages, e.g., [15, 23, 20, 26]. A number of generalisations of the theory have been proposed, notably to multi-party session types [22]. Multi-party session types have a close resemblance to choreographies as found in standards for business process modelling languages such as BPMN [27] and WS-CDL, and have been argued in theory to be able to provide typed BPMN processes [12].

Behavioral types usually furnish safety guarantees, notably progress and lock-freedom [5, 1, 7, 14, 33. In contrast, in this paper we extend binary session types to allow specification of liveness - the property of a process eventually "doing something good". Liveness properties are usually verified by model-checking techniques [9, 2, 6, requiring a state-space exploration. In the present paper we show that a fundamental class of liveness properties, so-called request-response properties, can be dealt with by type rules, that is, without resorting to state-space exploration. As a consequence, we can deal statically with infinite state systems as exemplified below. Also, liveness properties specified in types can be understood and used as interface specifications and for compositional reasoning.

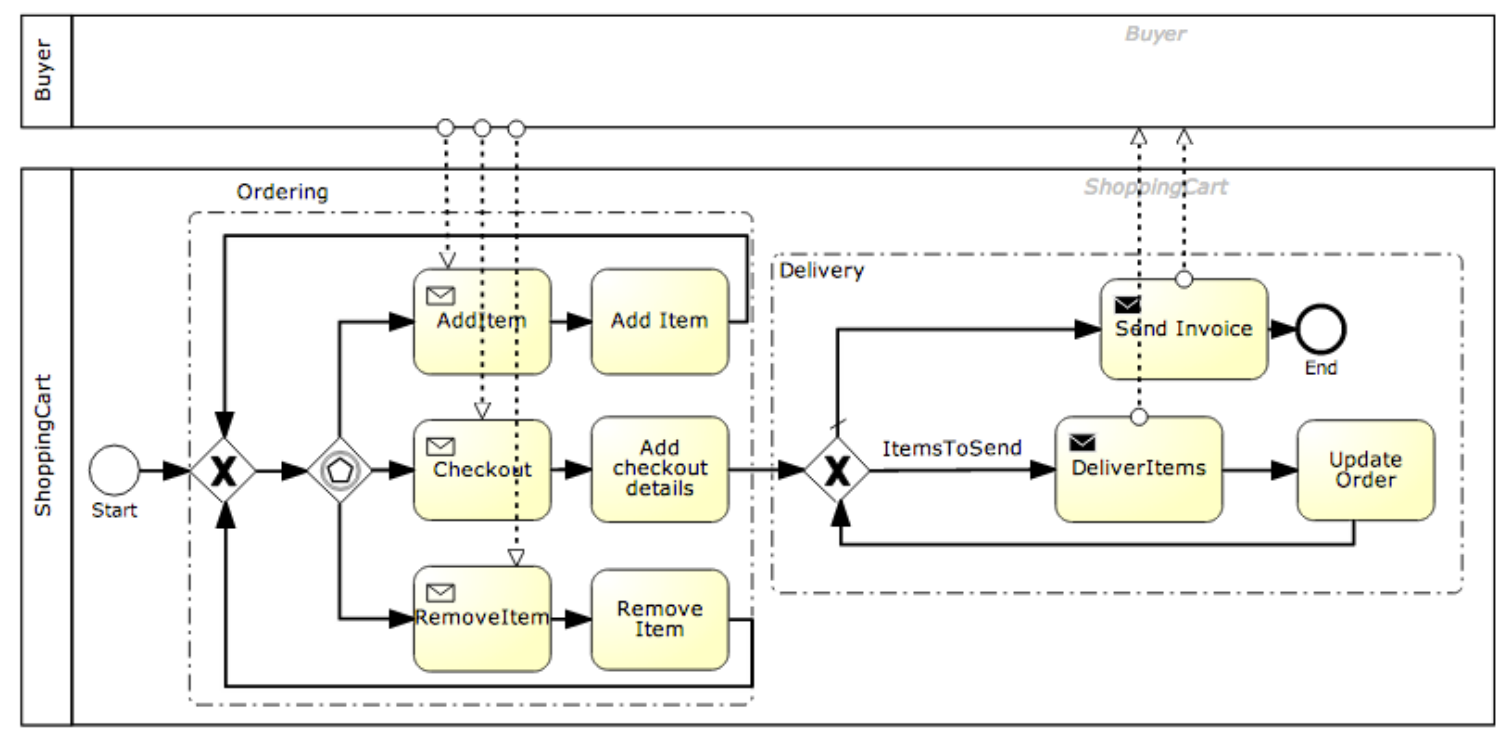

Figure A: A Potentially Non-live Shopping Cart BPMN Process

As an example, the above diagram contains two pools: The Buyer and the ShoppingCart. Only the latter specifies a process, which has two parts: Ordering and Delivery. Ordering is a loop starting with an event-based gateway, branching on the message received by the customer. If it is AddItem or RemoveItem, the appropriate item is added or removed from the order, whereafter the loop repeats. If it is Checkout, the loop is exited, and the Delivery phase commences. This phase is again a loop, delivering the ordered items and then sending the invoice to the buyer. 


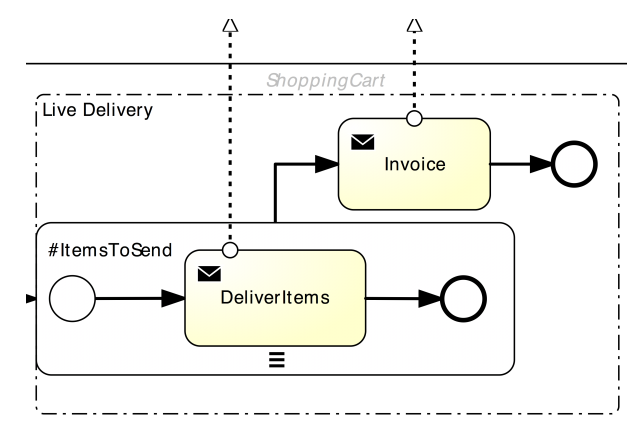

Figure B: Live delivery with MI Sub-Process

A buyer who wants to communicate safely with the Shopping Cart, must follow the protocol described above, and in particular must be able to receive an unbounded number of items before receiving the invoice. Writing AI, RI, CO, DI, and SI for the actions "Add Item", "Remove Item", "Checkout", "Deliver Items" and "Send Invoice"; we can describe this protocol from the point of view of the Shopping Cart with a session type:

$$
\mu t . \&\left\{\text { Al.?.t, RI.?.t, CO.?. } \mu t^{\prime} . \oplus\left\{\mathrm{DI} . \text {..t }{ }^{\prime}, \mathrm{SI} . \text {..end }\right\}\right\} \text {. }
$$

This session type can be regarded as a behavioral interface, specifying that the process first expects to receive either an Al (AddItem), RI (RemoveItem) or a CO (CheckOut) event. The two first events must be followed by a message (indicated by "?"), which in the implementation provides the item to be added or removed, after which the protocol returns to the initial state. The checkout event is followed by a message (again indicated by a "?") after which the protocol enters a new loop, either sending a DI (DeliverItem) event followed by a message (indicated by a "!") and repeating, or sending an SI (SendInvoice) event followed by a message (the invoice) and ending.

However, standard session types can not specify the very relevant liveness property, that a CO (checkout) event is eventually followed by a SI (send invoice) event. This is an example of a so-called request-response property: an action (the request) must be followed by a particular response. In this paper we conservatively extend binary session types to specify such request-response properties, and we show that this extension is strictly more expressive than standard session types. We do so by annotating the checkout selection in the type with the required response:

$$
\mu t . \&\left\{\mathrm{Al} . ? . t, \mathrm{RI} \text {.?.t, } \mathrm{CO}[\{\mathrm{SI}\}] . ? . \mu t^{\prime} . \oplus\left\{\mathrm{DI} . ! . t^{\prime}, \mathrm{SI} . \text { !.end }\right\}\right\} \text {. }
$$

Intuitively: "if CO is selected, then subsequently also SI must be selected."

Determining from the flow graph alone if this request-response property is guaranteed is in general not possible: Data values dictate whether the second loop terminates. However, we can remove this data-dependency by replacing the loop with a bounded iteration. In BPMN this can be realised by a Sequential Multiple Instance Sub-process, which sequentially executes a (run-time determined) number of instances of a sub-process. With this, we may re-define Delivery as in Fig. B, yielding a re-defined Shopping Cart process which has the request-response property.

In general, we need also be able to check processes where responses are requested within (potentially) infinite loops. The type system we present gives such guarantees, essentially by collecting all requested responses in a forward analysis, exploiting that potentially infinite 
loops can guarantee a particular response only if every path through the loop can; and that order (request-response vs response-request) is in this case irrelevant. We prove that, if the system is lock free, then the typing system indeed guarantees that all requested responses are eventually fulfilled. Lock-freedom is needed because, as is well known, collaborative processes with interleaved sessions may introduce dependency locks. Lock-freedom is well-studied for both $\pi$-calculus, e.g., [24, and binary session types [5, 1, 7, 14, 33, or may alternatively be achieved by resorting to global types $[22$.

In summary, our contributions are as follows.

- We extend binary session types with a notion of required response.

- We prove that this extension induces a strictly more expressive language class than standard session types.

- We give a typing system conservatively extending standard binary session types which gives the further guarantee that a lock-free well-typed process will, in any execution, provide all its required responses.

- We exemplify the use of these extended types to guarantee both safety and liveness properties for a non-trivial, infinite state collaborative process, which exhibits both possibly infinite looping and bounded iteration.

Related work. There is a vast amount of work on verification of collaborative processes. Most of the works take a model-checking approach, where the system under verification is represented as a kind of automaton or Petri Nets. An example that explicitly addresses collaborative business processes is [31], which however does not cover liveness properties. Live Sequence Charts (LSCs) [9] is a conservative extension of Message Sequence Charts allowing to distinguish possible (may) from required (must) behaviour, and thus the specification of liveness properties. LSCs can be mapped to symbolic timed automata [2] but relies as all model-checking approaches on abstraction techniques for reducing a large or even infinite state space to a tractable size. Here the work in [6] is interesting for the fact that the model-checking can be split on components. The work in [25] allows for model-checking of ML programs by a translation to higher-order recursion schemes. Interestingly, the model-checking problem is reduced to a type-checking problem, but rely on a technique for generation of a specific type system for the property of interest. In contrast, our approach is based on a single type system directly applicable for the process language at hand, where the (less general) liveness and safety properties of interest are specified as the type to be checked and can also be used as interface descriptions of processes. The fair subtyping of [28, the only work on session types addressing liveness we are aware of, details a liveness-preserving subtyping-relation for a session types-like CCS calculus. Here liveness is taken to mean the ability to always eventually output a special name, whereas in the present work, we consider the specification of fine-grained request-response liveness properties - "if something happens, something else must happen".

Overview of this paper. This article presents a full version of an extended abstract that appeared at FORTE '14 [11. The present paper includes the detailed definitions and explanations, many more examples, and complete proofs. In particular, the formal development for both basic correctness of the typing system in Section 4 as well as for the liveness results in Section 6 was mostly absent from the extended abstract; we believe these, in particular the latter, to be of independent interest. 
We proceed as follows. In Section 2 we define our calculus and its LTS-semantics. In Section 3 we extend binary session types with specification of request-response liveness properties, give transition semantics for types, and sketch a proof that the extended types induce a strictly larger class of languages than does standard types. In Section 4 we define exactly how types induce a notion of liveness on processes. In Section 5 we give our extended typing rules for sessions with responses and state its subject reduction result. In Section 6 we prove that the extended typing rules guarantees liveness for lock-free processes. Finally, in Section 7 we conclude.

We assume basic familiarity with $\pi$-calculus and binary session types, in particular the formulation of the latter in terms of polarised channels. A good introductory reference is [18; an extended discussion of the motivations for and ramifications of polarised channels is [34].

\section{Process Terms and Semantics}

Processes communicate only via named communication (session) channels by synchronizing send and receive actions or synchronizing select and branch events (as in standard session typed $\pi$-calculus). The session typing rules presented in the next section guarantees that there is always at most one active send and receive action for a given channel. To distinguish dual ends of communication channels, we employ polarised names [19, 34]: If $c$ is a channel name, $c^{+}$and $c^{-}$are the dual ends of the channel $c$. We call these polarised channel names, with "+" and "-" polarities. If $k$ is a polarised channel name, we write $\bar{k}$ for the dual polarised channel name, e.g., $\overline{c^{+}}=c^{-}$. The syntax of processes is given below.

Meta-variables:

$c \quad$ channel names

$p \quad$ polarities,+-

$k, h \quad$ polarised channel names $\left(c^{p}\right)$

$x \quad$ data variables

$v \quad$ data values, including natural numbers and true, false

$e \quad$ data expressions, including data variables and values

$l \quad$ selection labels

$X, Y$ process variables

Process syntax:

$$
\begin{aligned}
P::= & k !\langle e\rangle . P|k ?(x) . P| k ! l . P\left|k ?\left\{l_{i} . P_{i}\right\}_{i \in I}\right| \mathbf{0}|P| Q \\
& |\operatorname{rec} X . P|\left(\operatorname{rec}^{e} X(i) . P ; Q\right)|X[\tilde{k}]| \text { if } e \text { then } P \text { else } Q
\end{aligned}
$$

The first four process constructors are for taking part in a communication. These are standard for session typed $\pi$-calculi, except that for simplicity of presentation, we only allow data to be sent (see Section 7). The process $k !\langle e\rangle . P$ sends data $v$ over channel $k$ when $e \Downarrow v$, and proceeds as $P$. Dually, $k ?(x) . P$ receives a data value over channel $k$ and substitutes it for the $x$ binding in $P$. A branch process $k$ ? $\left\{l_{i} . P_{i}\right\}_{i \in I}$ offers a choice between labels $l_{i}$, proceeding to $P_{i}$ if the $i$ 'th label is chosen. The process $\mathbf{0}$ is the standard inactive process (termination), and $P \mid Q$ is the parallel composition of processes $P$ and $Q$.

Recursion comes in two forms: a general, potentially non-terminating recursion rec $X . P$, where $X$ binds in $P$; and a primitive recursion, guaranteed to terminate, with syntax $\left(\operatorname{rec}^{e} X(i) . P ; Q\right)$. The latter process, when $e$ evaluates to 0 , evolves to $Q$; when $e$ evalutes 
to $n+1$, it evolves to $P\{n / i\}\left\{\left(\operatorname{rec}^{e-1} X(i) . P ; Q\right)\right\}$, i.e., the process becomes $P$ with $n$ substituted for $i$, and the same process except for a decreased $e$ substituted for the process variable $X$. We assume the following conventions:

In $\left(\operatorname{rec}^{e} X(i) . P ; Q\right), \mathbf{0}$ does not occur in $P$.

In $\left(\operatorname{rec}^{e} X(i) . P ; Q\right)$, no process variable but $X$ occurs free in $P$.

In $\left(\operatorname{rec}^{e} X(i) . P ; Q\right)$, there is no sub-term $\operatorname{rec} Y . R$ or $\left(\operatorname{rec}^{e} Y(i) . R ; S\right)$ in $P$.

In $\left(\operatorname{rec}^{e} X(i) . P ; Q\right)$, there is no sub-term $R \mid S$ of $P$.

These conventions ensure that the process $\left(\operatorname{rec}^{e} X(i) . P ; Q\right)$ will eventually terminate the loop and execute $Q$. Process variables $X[\tilde{k}]$ mention the channel names $\tilde{k}$ active at unfolding time for technical reasons.

We define the free polarised names $\mathrm{fn}(P)$ of a process $P$ as usual, with $\mathrm{fn}(X[\tilde{k}])=\tilde{k}$; substitution of process variables from $X[\tilde{k}]\{P / X\}=P$; and finally value substitution $P\{v / x\}$ in the obvious way, e.g., $k !\langle e\rangle . P\{v / x\}=k !\langle e\{v / x\}\rangle .(P\{v / x\})$. Variable substitution can never affect channels.

Example 2.1. We now show how to model the example BPMN process given in the introduction. To illustrate the possibility of type checking infinite state systems, we use a persistent data object represented by a process $\operatorname{DATA}(o)$ communicating on a session channel $o$.

$$
\operatorname{DATA}(o)=\operatorname{rec} X . o^{+} ?(x) \cdot \operatorname{rec} Y . o^{+} ?\left\{\begin{array}{l}
\text { read. } o^{+} !\langle x\rangle \cdot Y\left[o^{+}\right] \\
\text {write. } X\left[o^{+}\right] \\
\text {quit. } \mathbf{0}
\end{array}\right.
$$

After having received its initial value, this process repeatedly accepts commands read and write on the session channel $o$ for respectively reading and writing its value, or the command quit for discarding the data object.

To make examples more readable, we employ the following shorthands. We write $\operatorname{init}(o, v) . P$ for $o^{-} !\langle v\rangle . P$, which initializes the data object; we write free $o . P$ for $o^{-}$!quit. $P$, the process which terminates the data object session; we write read $o(x) . P$ for $o^{-}$!read. $o^{-} ?(x) . P$, the process which loads the value of the data object $o$ into the process-local variable $x$; and finally, we write $o:=e . P$ for $o^{-}$!write. $o^{-} !\langle e\rangle . P$, the process which sets the value of the data-object $o$.

The shopping cart process can then be modelled as

$$
P(Q)=\operatorname{DATA}(o) \mid \operatorname{init}(o, \epsilon) \cdot \operatorname{rec} X . k\left\{\begin{array}{l}
\text { Al. } k ?(x) \cdot \operatorname{read} o(y) \cdot o:=\operatorname{add}(y, x) \cdot X\left[k o^{-}\right] \\
\text {RI. } k ?(x) \cdot \operatorname{read} o(y) \cdot o:=\operatorname{rem}(y, x) \cdot X\left[k o^{-}\right] \\
\text {CO. } k ?(x) \cdot \operatorname{read} o(y) \cdot o:=a d d(y, x) \cdot Q
\end{array}\right.
$$

Here $k$ is the session channel shared with the customer and $o$ is the session channel for communicating with the data object modelling order data. We assume our expression language has suitable operators "add" and "rem" for adding and removing items from the order. Finally, the process $Q$ is a stand-in for either the (non live) delivery part of the BPMN process in Fig. A or the live delivery part shown in Fig. B. The non-live delivery loop can be represented by the process

$$
\begin{array}{ll}
D_{0}=\operatorname{rec} Y . \operatorname{read} o(y) . \text { if } n(y)>0 & \begin{array}{l}
\text { then } k ! \mathrm{DI} . k !\langle n \operatorname{ext}(y)\rangle . o:=\operatorname{update}(y) . Y\left[k o^{-}\right] \\
\text {else } k ! \text { SI. } k !\langle\operatorname{inv}(y)\rangle . \text { free } o .0
\end{array}
\end{array}
$$


where $n(y)$ is the integer expression computing from the order $y$ the number of items to send, next $(y)$, update $(y)$ and $i n v(y)$ are, respectively, the next item(s) to be sent; an update of the order to mark that these items have indeed been sent; and the invoice for the order. Whether this process terminates depends on the data operations. Using instead bounded iteration, live delivery becomes:

$$
\begin{aligned}
& D=\operatorname{read} o(y) \cdot\left(\operatorname{rec}^{n(y)} Y(i) .\right. \\
& k ! \text { DI.read } o(y) \cdot k !\langle\operatorname{pickitem}(y, i)\rangle \cdot Y\left[k o^{-}\right] ; \\
& k ! S I . \operatorname{read} o(y) . k !\langle i n v(y)\rangle . \text { free } o .0)
\end{aligned}
$$

(The second line is the body of the loop; the third line is the continuation.) Here pickitem $(y, i)$ is the expression extracting the $i$ th item from the order $y$.

Transition Semantics. We give a labelled transition semantics in Fig C. We assume a total evaluation relation $e \Downarrow v$; note the absence of a structural congruence. Transition labels for processes are on one of the following forms.

$$
\lambda::=k ! v|k ? v| k \oplus l|k \& l| \tau \mid \tau: l
$$

We assume $\tau$ is neither a channel nor a polarised channel. Define $\operatorname{subj}(k ! v)=\operatorname{subj}(k ? v)=$ $\operatorname{subj}(k \& l)=\operatorname{subj}(k \oplus l)=k$ and $\operatorname{subj}(\tau)=\operatorname{subj}(\tau: l)=\tau$, and define as a technical convenience $\bar{\tau}=\tau$. We use these rules along with symmetric rules for [CfPARL] and C-Com $1 / 2]$.

Compared to standard CCS or $\pi$ semantics, there are two significant changes: (1) In the [C-PARL], a transition $\lambda$ of $P$ is not preserved by parallel composition if the co-channel of the subject of $\lambda$ is in $P^{\prime}$; and (2) in prefix rules, the co-name of the subject cannot appear in the continuation. We impose (1) because if the co-channel of the subject of $\lambda$ is in $P^{\prime}$, then intuitively the process $P \mid P^{\prime}$ already contains both sides of that session, and so does not offer synchronisation on that session to the environment, only to $P^{\prime}$. For instance, the process

$$
c^{+} !\langle v\rangle \cdot Q \mid c^{-} ?(x) \cdot R
$$

does not have a transition $c^{+} !\langle v\rangle \cdot Q\left|c^{-} ?(x) \cdot R \stackrel{c^{+} ! v}{\longrightarrow} Q\right| c^{-} ?(x) . R$. Such a transition would be useless: no environment $U$ able to receive on $c^{-}$could be put in parallel with $P$ and form a well-typed process, because both $U$ and $c^{-} ?(d) . R$ would contain the name $c^{-}$free. The reason for (2) is similar: If a process $k !\langle e\rangle \cdot P \stackrel{k ! v}{\longrightarrow} P$, and $P$ contains $\bar{k}$, again no well-typed environment for that process can contain $\bar{k}$. For (2), the side-condition could have been expressed as a well-formedness on syntax in the present setting; however, anticipating a future extension of the formalism to encompass also delegation, we have chosen the present condition on semantics instead.

In recent papers [32, 10, 33, session types have been presented not with polarised names, but rather with seemingly disparate names, connected by a new-name operator, e.g., one writes $(\nu x y)(x ! l . \mid y ?\{l .0\})$ to form a session with endpoints $x, y$. This latter formulation - while elegant for reduction semantics - is not viable for the present transition semantics. Without the ability to recognise the two ends $c^{+}, c^{-}$of a polarised channels as either end of a session, we cannot express the rules [C-PAR] nor [C-COM].

Lemma 2.2. If $P \stackrel{\lambda}{\rightarrow} Q$ then $\overline{\operatorname{subj}(\lambda)} \notin \mathrm{fn}(Q)$.

Proof. Straightforward induction on the derivation of the transition. 


$$
\begin{aligned}
& \text { [C-OUT] } \frac{e \Downarrow v}{k !\langle e\rangle \cdot P \stackrel{k ! v}{\longrightarrow} P} \bar{k} \notin \mathrm{fn}(P) \quad \text { [C-IN] } \frac{}{k ?(x) \cdot P \stackrel{k ? v}{\longrightarrow} P\{v / x\}} \bar{k} \notin \mathrm{fn}(P) \\
& \text { [C-SEL] } \underset{k ! l . P \stackrel{k \oplus l}{\longrightarrow} P}{\bar{k}} \notin \mathrm{fn}(P) \quad \text { Cf-BRA] } \underset{k ?\left\{l_{i} \cdot P_{i}\right\}_{i \in I} \stackrel{k \& l_{i}}{\longrightarrow} P_{i}}{k} \notin \mathrm{fn}\left(P_{i}\right) \\
& \text { [C.PARL] } \frac{P \stackrel{\lambda}{\rightarrow} Q}{P\left|P^{\prime} \stackrel{\lambda}{\rightarrow} Q\right| P^{\prime}} \quad \overline{\operatorname{subj}(\lambda)} \notin \mathrm{fn}\left(P^{\prime}\right) \\
& \text { [Cf-Com } 1] \quad \frac{P \stackrel{\bar{k} ! v}{\longrightarrow} P^{\prime} \quad Q \stackrel{k ? v}{\rightarrow} Q^{\prime}}{P\left|Q \stackrel{\tau}{\rightarrow} P^{\prime}\right| Q^{\prime}} \quad \text { [C-Com2] } \frac{P \stackrel{\bar{k} \oplus l}{\longrightarrow} P^{\prime} \quad Q \stackrel{k \& l}{\longrightarrow} Q^{\prime}}{P\left|Q \stackrel{\tau: l}{\longrightarrow} P^{\prime}\right| Q^{\prime}} \\
& \text { [C-REC] } \frac{P\{\operatorname{rec} X . P / X\} \stackrel{\lambda}{\rightarrow} Q}{\operatorname{rec} X . P \stackrel{\lambda}{\rightarrow} Q} \quad \text { [C.PREC0] } \frac{e \Downarrow 0 \quad Q \stackrel{\lambda}{\rightarrow} R}{\left(\operatorname{rec}^{e} X(i) . P ; Q\right) \stackrel{\lambda}{\rightarrow} R} \\
& \text { [C-PRECN] } \frac{e \Downarrow n+1 \quad P\{n / i\}\left\{\left(\operatorname{rec}^{n} X(i) \cdot P ; Q\right) / X\right\} \stackrel{\lambda}{\rightarrow} R}{\left(\operatorname{rec}^{e} X(i) \cdot P ; Q\right) \stackrel{\lambda}{\rightarrow} R} \\
& \text { [C]CONDT] } \frac{e \Downarrow \text { true } P \stackrel{\lambda}{\rightarrow} P^{\prime}}{\text { if } e \text { then } P \text { else } Q \stackrel{\lambda}{\rightarrow} P^{\prime}} \quad \text { [C-CondF] } \frac{e \Downarrow \text { false } Q \stackrel{\lambda}{\rightarrow} Q^{\prime}}{\text { if } e \text { then } P \text { else } Q \stackrel{\lambda}{\rightarrow} Q^{\prime}}
\end{aligned}
$$

Figure C: Transition semantics for terms

\section{Session Types with Responses}

In this section, we generalise binary session types to session types with responses. In addition to providing the standard communication safety properties, these also allow us to specify request-response liveness properties.

Compared to standard session types, we do not consider delegation (name passing). Firstly, as illustrated by our example calculus, the types are already expressive enough to cover a non-trivial subset of collaborative processes. Secondly, as we show in the end of the section, session types with responses are already strictly more expressive than standard session types with respect to the languages they can express. Thus, as we also address in Section 7, admitting delegation and answering the open question about how response obligations can be safely exchanged with the environment, is an interesting direction for future work which is beyond the scope of the present paper.

We first define request-response liveness in the abstract. In general, we shall take it to be the property that "a request is eventually followed by a response". For now, we will not concern ourselves exactly what "requests" and "responses" are or what

it means for a response to fulfil a request. 
Definition 3.1. A request-response structure is a tuple $(A, R$, req, res) where $A$ is a set of actions, $R$ is a set of responses, and req : $A \rightarrow R$ and res : $A \rightarrow R$ are maps defining the set of responses that an action requests and performs, respectively.

Notation. In this setting, response liveness is naturally a property of sequences. We write $\epsilon$ for the empty sequence, we let $\phi, \psi$ range over finite sequences, and we let $\alpha, \beta, \gamma$ range over finite or infinite sequences. We write sequence concatenation by juxtaposition, i.e., $\phi \alpha$.

Definition 3.2. Suppose $(A, R$, req, res) is a request-response structure and $\alpha$ a sequence over $A$. Then the responses $\operatorname{res}(\alpha)$ of $\alpha$ is defined by $\operatorname{res}(\alpha)=\cup\{\operatorname{res}(a) \mid \exists \varphi, \beta . \alpha=\varphi a \beta\}$. Moreover, $\alpha$ is live iff $\alpha=\phi a \beta \Longrightarrow \operatorname{req}(a) \subseteq \operatorname{res}(\beta)$.

Notation. We shall be specially interested in liveness of sequences of transitions. A finite transition sequence of length $n$ is a pair of sequences $\left(s_{i}\right)_{i<n}$ and $\left(t_{i}\right)_{i<n-1}$ s.t. $s_{i} \stackrel{t_{i}}{\rightarrow} s_{i+1}$ for $i<n$. An infinite transition sequence is a pair of sequences $\left(s_{i}\right)_{i \in \mathbb{N}}$ and $\left(t_{i}\right)_{i \in \mathbb{N}}$ s.t. $s_{i} \stackrel{t_{i}}{\rightarrow} s_{i+1}$. A finite or infinite transition sequence of a state $s$ is a finite or infinite transition sequence with $s_{1}=s$. We write $\left(s_{i}, t_{i}\right)_{i \in \mathbb{N}}$ for infinite sequences and $\left(\left(s_{i}, t_{i}\right)_{i<n}, s_{n}\right)$ for finite sequences, giving the final state explicitly. Slightly abusing notation, we sometimes write $\left(s_{i}, t_{i}\right)_{i \in I}$ or even just $\left(s_{i}, t_{i}\right)$ for a finite or infinite transition sequence, saying that it is a transition sequence of $s_{1}$ over $I$.

Definition 3.3 (LTS with responses). Let $(S, L, \rightarrow)$ be an LTS. When the set of labels $L$ is the set of actions of a request-response structure $\rho$, we say that $(S, L, \rightarrow, \rho)$ is an LTS with responses, and that a transition sequence of this LTS is live when its underlying sequence of labels is.

Next, we present the syntax of types.

$$
\begin{array}{ll}
\mathcal{L} & \text { a countably infinite set of labels } \\
l & \text { ranges over } \mathcal{L} \\
L & \text { ranges over } \mathcal{P}(\mathcal{L}) \\
S, T & ::=\&\left\{l_{i}\left[L_{i}\right] . T_{i}\right\}_{i \in I}\left|\oplus\left\{l_{i}\left[L_{i}\right] . T_{i}\right\}_{i \in I}\right| \text { I.T } \mid \text { ?.T | } \mu t . T|t| \text { end }
\end{array}
$$

By convention, the $l_{i}$ in each $\&\left\{l_{i}\left[L_{i}\right] . T_{i}\right\}_{i \in I}$ resp. $\oplus\left\{l_{i}\left[L_{i}\right] . T_{i}\right\}_{i \in I}$ are distinct.

A session type is a (possibly infinite) tree of actions permitted for one partner of a two-party communication. The type $\&\left\{l_{i}\left[L_{i}\right] \cdot T_{i}\right\}_{i \in I}$, called branch, is the type of offering a choice between different continuations. If the partner chooses the label $l_{i}$, the session proceeds as $T_{i}$. Compared to standard session types, making the choice $l_{i}$ also requests a subsequent response on every label mentioned in the set of labels $L_{i}$; we formalise this in the notion of responsive trace below. Dual to branch is select $\oplus\left\{l_{i}\left[L_{i}\right] . T_{i}\right\}_{i \in I}$ : the type of making a choice between different continuations. Like branch, making a choice $l_{i}$ requests every label in $L_{i}$ as future responses. The type !.T and ?.T are the types of sending and receiving data values. As mentioned above, channels cannot be communicated. Also, we have deliberately omitted types of values (e.g. integers, strings, booleans) being sent, since this can be trivially added and we want to focus on the behavioural aspects of the types. Finally, session types with responses include recursive types. We take the equi-recursive view, identifying a type $T$ and its unfolding into a potentially infinite tree. We define the 
Type transition labels: $\quad \rho::=!|?| \& l[L] \mid \oplus l[L]$ Type transition label duality: $\quad ! \bowtie ? \quad$ and $\quad \& l[L] \bowtie \oplus l\left[L^{\prime}\right]$

$$
\begin{aligned}
& \text { [D-Out] } \overline{! . T \stackrel{!}{\rightarrow} T} \quad \overline{? . T \stackrel{?}{\rightarrow} T} \quad \text { [D-IN] } \\
& \text { [D.BRA } \frac{i \in I}{\&\left\{l_{i}\left[L_{i}\right] . T_{i}\right\}_{i \in I} \stackrel{\& l_{i}\left[L_{i}\right]}{\longrightarrow} T_{i}} \quad \frac{i \in I}{\oplus\left\{l_{i}\left[L_{i}\right] . T_{i}\right\}_{i \in I} \stackrel{\oplus l_{i}\left[L_{i}\right]}{\longrightarrow} T_{i}} \quad \text { D.SEL] }
\end{aligned}
$$

Figure D: Transitions of types (1)

central notion of duality between types as the symmetric relation induced coinductively by the following rules.

$$
\overline{\text { end } \bowtie \text { end }} \quad \frac{T \bowtie T^{\prime}}{! . T \bowtie ? . T^{\prime}} \quad \frac{T_{i} \bowtie T_{i}^{\prime} \quad J \subseteq I}{\&\left\{l_{i}\left[L_{i}\right] \cdot T_{i}\right\}_{i \in I} \bowtie \oplus\left\{l_{j}\left[L_{j}^{\prime}\right] . T_{j}^{\prime}\right\}_{j \in J}}
$$

The first rule says that dual processes agree on when communication ends; the second that if a process sends a message, its dual must receive; and the third says that if one process offers a branch, its dual must choose among the offered choices. However, required responses do not need to match: the two participants in a session need not agree on the notion of liveness for the collaborative session.

Example 3.4. Recall from Ex. 2.1 the processes DATA $(o)$ encoding data-object and $P(D)$ encoding the (live) shopping-cart process. The former treats the channel $o$ as

$$
T_{D}=\mu t . ? . \mu s . \&\{\text { read.!.s, write.t, quit.end }\} .
$$

The latter treats its channel $k$ to the buyer as

$$
T_{P}=\mu t . \&\left\{\mathrm{Al} . \text { ?.t, RI.?.t, } \mathrm{CO}[\{\mathrm{SI}\}] . ? . \mu t^{\prime} . \oplus\left\{\mathrm{DI} . . . t^{\prime}, \mathrm{SI} . . . e n d\right\}\right\} .
$$

To illustrate both responses in unbounded recursion and duality of disparate responses, note that the $P(D)$ actually treats its data object channel $o^{-}$according to the type $T_{E}=\mu t . ! . \mu s . \oplus\{$ read.?.s, write $[\{\mathrm{read}\}] . t$, quit.end $\}$, i.e., every write is eventually followed by a read. However, $T_{D} \bowtie T_{E}$ : the types $T_{E}$ and $T_{D}$ are nonetheless dual.

Having defined the syntax of session types with responses, we proceed to give their semantics. The meaning of a session type is the possible sequences of communication actions it allows, requiring that pending responses eventually be done. Formally, we equip session types with a labeled transition semantics in Fig. D.

We emphasise that under the equi-recursive view of session types, the transition system of a recursive type $T$ may in general be infinite.

Taking actions $A$ to be the set of labels ranged over by $\rho$, and recalling that $\mathcal{L}$ is our universe of labels for branch/select, we obtain a request-response structure $(A, \mathcal{P}(\mathcal{L})$, req, res) with the latter two operators defined as follows.

$$
\begin{array}{ll}
\operatorname{res}(!)=\operatorname{res}(?)=\emptyset & \operatorname{res}(\& l[L])=\operatorname{res}(\oplus l[L])=\{l\} \\
\operatorname{req}(!)=\operatorname{req}(?)=\emptyset & \operatorname{req}(\& l[L])=\operatorname{req}(\oplus l[L])=L
\end{array}
$$


In the right-hand column, selecting a label $l$ performs the response $l$; pending responses $L$ associated with that label are conversely requested. The LTS of Fig. D is thus one with responses, and we may speak of its transition sequences being live or not.

Definition 3.5. Let $T$ be a type. We define:

(1) The traces $\operatorname{tr}(T)=\left\{\left(\rho_{i}\right)_{i \in I} \mid\left(T_{i}, \rho_{i}\right)_{i \in I}\right.$ transition sequence of $\left.T\right\}$

(2) The responsive traces $\operatorname{tr}_{R}(T)=\{\alpha \in \operatorname{tr}(T) \mid \alpha$ live $\}$.

That is, in the responsive traces any request is followed by a response.

Definition 3.6. A type $T$ is a standard session type if it requests no responses, that is, every occurrence of $L$ in it is has $L=\emptyset$. Define an operator sel(-) as follows, lifting it pointwise to sequences.

$$
\operatorname{sel}(!)=\operatorname{sel}(?)=\epsilon \quad \operatorname{sel}(\& l[L])=\operatorname{sel}(\oplus l[L])=l
$$

We then define:

(1) The selection traces $\operatorname{str}(T)=\{\operatorname{sel}(\alpha) \mid \alpha \in \operatorname{tr}(T)\}$

(2) The responsive selection traces $\operatorname{str}_{R}(T)=\left\{\operatorname{sel}(\alpha) \mid \alpha \in \operatorname{tr}_{R}(T)\right\}$.

(3) The languages of standard session types

$\mathcal{T}=\{\operatorname{str}(T) \mid T$ is a standard session type $\}$.

(4) The languages of responsive session types

$\mathcal{R}=\left\{\operatorname{str}_{R}(T) \mid T\right.$ is a session type with responses $\}$.

That is, we compare standard session types and session types of responses by considering the sequences of branch/select labels they admit. This follows recent work on multi-party session types and automata [12, 13].

A fine point: because the sel(-) map is lifted pointwise and maps "no selection" to the empty sequence $\epsilon$, this definition of languages is oblivious to send and receive. E.g, if $\phi_{S}, \psi_{T}$ are the unique traces of the two types $S=$ !. $\oplus l . \oplus l^{\prime}$.end and $T=\oplus l . ? . \oplus l^{\prime}$.end, then $\operatorname{sel}\left(\phi_{S}\right)=\operatorname{sel}\left(\psi_{T}\right)=l l^{\prime}$. We formalise this insight in the following lemma.

Lemma 3.7. Let $T$ be a standard session type. There exists a session type $T^{\prime}$ with no occurrences of send !.T or receive ?.T s.t. $\operatorname{str}(T)=\operatorname{str}\left(T^{\prime}\right)$.

Example 3.8. The type $T_{P}$ of Example 3.4 has (amongst others) the two selection traces: $t=\mathrm{AICODIDISI}$ and $u=\mathrm{AICO} \mathrm{DIDIDI} \cdots$. Of these, only $t$ is responsive; $u$ is not, since it never selects $\mathrm{SI}$ as required by its $\mathrm{CO}$ action. That is, $t, u \in \operatorname{str}\left(T_{P}\right)$ and $t \in \operatorname{str}_{R}\left(T_{P}\right)$, but $u \notin \operatorname{str}_{R}\left(T_{P}\right)$.

Lemma 3.9 (Session types with responses are deterministic). (1). If $T \stackrel{\rho}{\rightarrow}$ and $T \stackrel{\rho^{\prime}}{\rightarrow}$ and $\operatorname{sel}(\rho)=\operatorname{sel}\left(\rho^{\prime}\right) \neq \epsilon$, then $\rho=\rho^{\prime}$. (2). Consider equally long finite transition sequences $\left(\left(T_{i}, \rho_{i}\right)_{i<n}, T_{n}\right)$ and $\left(\left(S_{i}, \rho_{i}^{\prime}\right)_{i<n}, S_{n}\right)$. If $T_{1}=S_{1}$ and for each $i<n \rho_{i}=\rho_{i}^{\prime}$, then also $T_{i}=S_{i}$ for each $i \leq n$.

Proof. (1). Immediate from the convention that each label in a branch or selected is distinct. (2). By induction on $n$. The base case is trivial. For $n=k+1$ we have by the induction hypothesis $T_{k}=S_{k}$. By convention, each label in a branch or select is distinct, so there is at most one $S$ with $T_{k} \stackrel{\rho_{k}}{\rightarrow} S$. But then $S=T_{k+1}=S_{k+1}$. 
Theorem 3.10. The language of session types with responses $\mathcal{R}$ is strictly more expressive than that of standard session types $\mathcal{T}$; that is, $\mathcal{T} \subset \mathcal{R}$.

Proof. The non-strict inclusion is immediate by definition; it remains to prove it strict. Consider the following session type with responses, $T$.

$$
T=\mu t . \oplus\left\{\begin{array}{l}
a[b] . t \\
b[a] . t
\end{array}\right.
$$

We shall prove that $\operatorname{str}_{R}(T) \notin \mathcal{T}$. Suppose not; then there exists a session type $S$ with

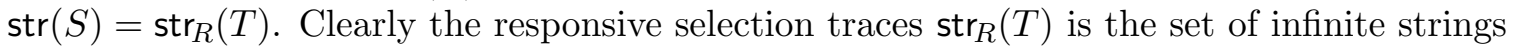
over the alphabet $\{a, b\}$ where both $a, b$ occur infinitely often. It follows that for all $k>0$, the string $a^{k}$ is a prefix of an infinite string in $\operatorname{str}_{R}(T)$. We have assumed $\operatorname{str}(S)=\operatorname{str}_{R}(T)$, so each $a^{k}$ must also be a prefix of an infinite string in $\operatorname{tr}(S)$. By Lemma 3.7, we may assume $S$ has no occurrences of send or receive, and so for each $k$ there is a transition sequence $\left(\left(S_{i}^{k}, \rho_{i}^{k}\right)_{i<k}, S_{k}^{k}\right)$ with $S_{1}^{k}=S$ and sel $\left(\rho_{i}^{k}\right)=a$. By induction on $k$ using Lemma 3.9 , we find that $\rho_{i}^{k}=\rho_{i}^{k+1}$ and $S_{i}^{k}=S_{i}^{k+1}$ when $i \leq k$. It follows that $S_{i}^{i}=S_{i}^{i+1} \stackrel{\rho_{i}}{\longrightarrow} S_{i+1}^{i+1}$ when $i+1 \leq k$, and so $\left(S_{i}^{i}, \rho_{i}\right)_{i \in \mathbb{N}}$ is an infinite transition sequence with $S_{1}^{1}=S$. But then $\left(\operatorname{sel}\left(\rho_{i}\right)\right)_{i \in \mathbb{N}}=a^{\omega} \in \operatorname{str}(S)$ while clearly not in $\operatorname{str}_{R}(T)$, contradicting $\operatorname{str}_{R}(T)=\operatorname{str}(S)$.

\section{SEssion TyPing}

Recall the standard type system for session types, presented in Fig. E with the obvious extension for primitive recursion. In this judgement, $\Theta$ takes process variables to session type environments; in turn, a session typing environment $\Delta$ is a finite partial map from channel names and polarised channel names to types.

$$
\begin{aligned}
\Theta & ::=\epsilon \mid \Theta, X: \Delta \\
\Delta & ::=\epsilon \mid k: T
\end{aligned}
$$

We write $\Delta, \Delta^{\prime}$ for the union of $\Delta$ and $\Delta^{\prime}$, defined when their domains are disjoint. We say $\Delta$ is completed if $\Delta(k)=$ end when defined; it is balanced if $k: T, \bar{k}: U \in \Delta$ implies $T \bowtie U$.

We generalise transitions of types (Fig. D) to session typing environments in Fig. $\mathrm{F}$, with transitions ranged over by $\delta$ as follows; recall that $\rho$ is a type transition label.

$$
\delta::=\tau|\tau: l, L| k: \rho
$$

We define $\operatorname{subj}(k: \rho)=k$ and $\operatorname{subj}(\tau: l, L)=\operatorname{subj}(\tau)=\tau$. We lift $\operatorname{sel}(-), \operatorname{req}(-)$, and $\operatorname{res}(-)$ to actions $\delta$ as follows.

$$
\begin{array}{lll}
\operatorname{sel}(\tau)=\epsilon & \operatorname{sel}(k: \rho)=\operatorname{sel}(\rho) & \operatorname{sel}(\tau: l, L)=l \\
\operatorname{req}(\tau)=\emptyset & \operatorname{req}(\tau: \rho)=\operatorname{req}(\rho) & \operatorname{req}(\tau: l, L)=L \\
\operatorname{res}(\tau)=\emptyset & \operatorname{res}(k: \rho)=\operatorname{res}(\rho) & \operatorname{res}(k: l, L)=\{l\}
\end{array}
$$

The type environment transition is thus an LTS with responses, and we may speak of its transition sequences being live.

Definition 4.1. We define a binary relation on type transition labels $\delta$ and transition labels $\lambda$, written $\delta \simeq \lambda$, as follows.

$$
\begin{aligned}
& \tau \simeq \tau \quad k: \& l[L] \simeq k \& l \quad k: ! \simeq k ! v \\
& \tau: l, L \simeq \tau: l \quad k: \oplus l[L] \simeq k \oplus l \quad k: ? \simeq k ? x
\end{aligned}
$$




$$
\begin{aligned}
& \text { [E-Out] } \frac{\Theta \vdash_{\text {std }} P \triangleright \Delta, k: T}{\Theta \vdash_{\text {std }} k !\langle e\rangle \cdot P \triangleright \Delta, k: ! . T} \quad \frac{\Theta \vdash_{\text {std }} P \triangleright \Delta, k: T}{\Theta \vdash_{\text {std }} k ?(x) \cdot P \triangleright \Delta, k: ? . T} \quad \text { [E-IN] } \\
& \text { [E-BRA] } \frac{\forall i \in I: \quad \Theta \vdash_{\text {std }} P_{i} \triangleright \Delta, k: T_{i}}{\Theta \vdash_{\text {std }} k ?\left\{l_{i} . P_{i}\right\}_{i \in I} \triangleright \Delta, k: \&\left\{l_{i}\left[L_{i}\right] . T_{i}\right\}_{i \in I}} \\
& \text { [E-SEL] } \frac{\Theta \vdash_{\text {std }} P \triangleright \Delta, k: T_{j}}{\Theta \vdash_{\text {std }} k ! l_{j} . P \triangleright \Delta, k: \oplus\left\{l_{i}\left[L_{i}\right] \cdot T_{i}\right\}_{i \in I}} \quad(j \in I) \\
& \text { [E-PAR] } \quad \frac{\Theta \vdash_{\text {std }} P_{1} \triangleright \Delta_{1} \quad \Theta \vdash_{\text {std }} P_{2} \triangleright \Delta_{2}}{\Theta \vdash_{\text {std }} P_{1} \mid P_{2} \triangleright \Delta_{1}, \Delta_{2}} \quad \frac{\Delta \text { completed }}{\Theta \vdash_{\text {std }} \mathbf{0} \triangleright \Delta} \quad \text { [E-INACT] } \\
& \frac{\Theta, X: \Delta \vdash_{\text {std }} P \triangleright \Delta \quad \Theta \vdash_{\text {std }} Q \triangleright \Delta}{\Theta \vdash_{\text {std }}\left(\operatorname{rec}^{e} X(i) \cdot P ; Q\right) \triangleright \Delta} \quad[\mathrm{E}-\mathrm{RECP}] \\
& \text { [E-REC] } \frac{\Theta, X: \Delta \vdash_{\text {std }} P \triangleright \Delta}{\Theta \vdash_{\text {std }} \operatorname{rec} X . P \triangleright \Delta} \quad \frac{\operatorname{dom}(\Delta)=\tilde{k}}{\Theta, X: \Delta \vdash_{\text {std }} X[\tilde{k}] \triangleright \Delta} \quad \text { [E-VAR] } \\
& \text { [E-Cond }] \frac{\Theta \vdash_{\text {std }} P \triangleright \Delta \quad \Theta \vdash_{\text {std }} Q \triangleright \Delta}{\Theta \vdash_{\text {std }} \text { if } e \text { then } P \text { else } Q \triangleright \Delta}
\end{aligned}
$$

Figure E: Standard Session Typing System

$$
\left[\text { [F-LiFt] } \frac{T \stackrel{\rho}{\rightarrow} T^{\prime}}{k: T \stackrel{k: \rho}{\rightarrow} k: T^{\prime}} \quad \text { [F-PAR] } \frac{\Delta \stackrel{\delta}{\rightarrow} \Delta^{\prime}}{\Delta, \Delta^{\prime \prime} \stackrel{\delta}{\rightarrow} \Delta^{\prime}, \Delta^{\prime \prime}}\right.
$$

[F-Com1] $\frac{\Delta_{1} \stackrel{k: !}{\longrightarrow} \Delta_{1}^{\prime} \quad \Delta_{2} \stackrel{\bar{k}: ?}{\longrightarrow} \Delta_{2}^{\prime}}{\Delta_{1}, \Delta_{2} \stackrel{\tau}{\rightarrow} \Delta_{1}^{\prime}, \Delta_{2}^{\prime}} \quad$ [F-CoM2] $\frac{\Delta_{1} \stackrel{k: \oplus l[L]}{\longrightarrow} \Delta_{1}^{\prime} \quad \Delta_{2} \stackrel{\bar{k}: \& l\left[L^{\prime}\right]}{\longrightarrow} \Delta_{2}^{\prime}}{\Delta_{1}, \Delta_{2} \stackrel{\tau: l, L \cup L^{\prime}}{\longrightarrow} \Delta_{1}^{\prime}, \Delta_{2}^{\prime}}$

Figure F: Transitions of types (2)

Theorem 4.2 (Subject reduction). If $\Gamma \vdash_{\text {std }} P \triangleright \Delta$ and $P \stackrel{\lambda}{\rightarrow} Q$, then there exists $\delta \simeq \lambda$ s.t. $\Delta \stackrel{\delta}{\rightarrow} \Delta^{\prime}$ and $\Gamma \vdash_{\text {std }} Q \triangleright \Delta^{\prime}$.

The proof is in Appendix A.

Definition 4.3. The typed transition system is the transition system which has states $\Gamma \vdash_{\text {std }} P \triangleright \Delta$ and transitions $\Gamma \vdash_{\text {std }} P \triangleright \Delta \stackrel{\lambda, \delta}{\longrightarrow} \Gamma \vdash_{\text {std }} P^{\prime} \triangleright \Delta^{\prime}$ whenever there exist transitions $P \stackrel{\lambda}{\rightarrow} P^{\prime}$ and $\Delta \stackrel{\delta}{\rightarrow} \Delta^{\prime}$ with $\delta \simeq \lambda$. 
Example 4.4. Wrt. the standard session typing system, both of the processes $P\left(D_{0}\right)$ and $P(D)$ of Example 2.1 are typable wrt. the types we postulated for them in Example 3.4 . Specifically, we have $\cdot \vdash_{\text {std }} P\left(D_{0}\right) \triangleright k: T_{P}, o^{+}: T_{D}, o^{-}: \overline{T_{D}}$ and similarly for $P(D)$. The judgement means that the process $P(D)$ treats $k$ according to $T_{P}$ and the (two ends of) the data object according to $T_{D}$ and its syntactic dual $\overline{T_{D}}$. The standard session typing system of course does not act on our liveness annotations, and so does not care that $P\left(D_{0}\right)$ is not live.

For the subsequent development, we will need the following lemmas.

Lemma 4.5. If $\Delta \stackrel{\delta}{\rightarrow} \Delta^{\prime}$ then $\operatorname{dom}(\Delta)=\operatorname{dom}\left(\Delta^{\prime}\right)$.

Proof. Straightforward induction on the derivation of the transition.

Lemma 4.6. If $\Delta \stackrel{\delta}{\rightarrow} \Delta^{\prime}$ then either:

(1) $\delta=k: \rho$ and $\Delta=\Delta^{\prime \prime}, k: T$ and $\Delta^{\prime}=\Delta^{\prime \prime}, k: T^{\prime}$ and $T \stackrel{\rho}{\rightarrow} T^{\prime}$; or

(2) $\delta=\tau$ or $\delta=\tau: l, L$ and $\Delta=\Delta^{\prime \prime}, k: T, \bar{k}: S$ and $\Delta^{\prime}=\Delta^{\prime \prime}, k: T^{\prime}, \bar{k}: S^{\prime}$ where $T \stackrel{\rho}{\rightarrow} T^{\prime}$ and $S \stackrel{\rho^{\prime}}{\rightarrow} S^{\prime}$ and $\rho \bowtie \rho^{\prime}$.

Proof. Straightforward induction on the derivation of the transition.

Lemma 4.7. If $\Delta \stackrel{\delta}{\rightarrow} \Delta^{\prime}$ with $\Delta$ balanced and $\overline{\operatorname{subj}(\delta)} \notin \operatorname{dom}(\Delta)$, then also $\Delta^{\prime}$ balanced.

Proof. By induction on the derivation of the transition.

Case [F-LifT]. Trivial.

Case [F-PAR]. Suppose $\Delta, \Delta^{\prime \prime}$ balanced with $\overline{\operatorname{subj}(\delta)} \notin \operatorname{dom}\left(\Delta, \Delta^{\prime \prime}\right)$, and consider $k, \bar{k} \in$ $\operatorname{dom}\left(\Delta^{\prime}, \Delta^{\prime \prime}\right)$. If both are in $\operatorname{dom}\left(\Delta^{\prime \prime}\right)$, we are done. If both are in $\operatorname{dom}\left(\Delta^{\prime}\right)$ then by the Lemma 4.5 they are also in $\operatorname{dom}(\Delta)$. Then, because $\Delta, \Delta^{\prime \prime}$ balanced implies $\Delta$ balanced, we find by the induction hypothesis that also $\Delta^{\prime}$ balanced, whence $\left(\Delta^{\prime}, \Delta^{\prime \prime}\right)(k)=\Delta^{\prime}(k) \bowtie$ $\Delta^{\prime}(\bar{k})=\left(\Delta^{\prime}, \Delta^{\prime \prime}\right)(\bar{k})$. Finally, suppose wlog $k \in \operatorname{dom}\left(\Delta^{\prime}\right)$ and $\bar{k} \in \operatorname{dom}\left(\Delta^{\prime \prime}\right)$; we shall see that this is not possible. By Lemma 4.6 either

$$
\Delta=\Delta_{1}, k: T \stackrel{k: \rho}{\longrightarrow} \Delta_{1}, k: T^{\prime}=\Delta^{\prime} \quad \text { with } T \stackrel{\rho}{\rightarrow} T^{\prime},
$$

or

$$
\Delta=\Delta_{2}, k: T, \bar{k}: S \stackrel{\delta}{\rightarrow} \Delta_{2}, k: T^{\prime}, \bar{k}: S^{\prime}=\Delta^{\prime} \quad \text { with } T \stackrel{\rho}{\rightarrow} T^{\prime} \text { and } S \stackrel{\bar{\rho}}{\rightarrow} S^{\prime} .
$$

We consider these two possibilities in turn. It cannot be true that (4.1) holds, because by the assumption $\overline{\operatorname{subj}(\delta)} \notin \operatorname{dom}\left(\Delta, \Delta^{\prime \prime}\right)$ we must have $\bar{k}=\overline{\operatorname{subj}(\delta)} \notin \operatorname{dom}\left(\Delta^{\prime \prime}\right)$, contradicting $\bar{k} \in \operatorname{dom}\left(\Delta^{\prime \prime}\right)$. If instead (4.2) holds, then because $\bar{k} \in \operatorname{dom}\left(\Delta^{\prime \prime}\right)$ then $\Delta, \Delta^{\prime \prime}$ is not defined, contradicting the existence of the transition $\Delta, \Delta^{\prime \prime} \stackrel{\delta}{\rightarrow} \Delta^{\prime}, \Delta^{\prime \prime}$.

Case [F-Com1]. Suppose $\Delta_{1}, \Delta_{2} \stackrel{\tau}{\rightarrow} \Delta_{1}^{\prime}, \Delta_{2}^{\prime}$ with $\Delta_{1}, \Delta_{2}$ balanced. Using Lemma 4.6, and [F-Com1] we have transitions $\Delta_{1}=\Delta_{3}, k: S \stackrel{k: !}{\longrightarrow} \Delta_{3}, k: S^{\prime}=\Delta_{1}^{\prime}$ and $\Delta_{2}=\Delta_{4}, \bar{k}: T \stackrel{\bar{k}: ?}{\longrightarrow}$ $\Delta_{4}, \bar{k}: T^{\prime}=\Delta_{2}^{\prime}$, with $S \stackrel{!}{\rightarrow} S^{\prime}$ and $T \stackrel{?}{\rightarrow} T^{\prime}$. It follows that our original transition is on the form

$$
\Delta_{1}, \Delta_{2}=\Delta_{3}, k: S, \Delta_{4}, \bar{k}: T \stackrel{\tau}{\rightarrow} \Delta_{3}, k: S^{\prime}, \Delta_{4}, \bar{k}: T^{\prime}=\Delta_{1}^{\prime}, \Delta_{2}^{\prime} .
$$

Because $\Delta_{1}, \Delta_{2}$ balanced then also $\Delta_{3}, \Delta_{4}$ is, and so $\Delta_{1}^{\prime}, \Delta_{2}^{\prime}=\Delta_{3}, k: S^{\prime}, \Delta_{4}, \bar{k}: T^{\prime}$ is balanced if $S^{\prime} \bowtie T^{\prime}$. But $S \stackrel{!}{\rightarrow} S^{\prime}$ implies $S=! . S^{\prime}$ and $T \stackrel{?}{\rightarrow} T^{\prime}$ implies $T=$ ?.T $T^{\prime}$. But we have !. $S^{\prime}=S \bowtie T=? . T^{\prime}$ by $\Delta_{1}, \Delta_{2}$ balanced, and so by definition $S^{\prime} \bowtie T^{\prime}$. 
Case [F-Com2]. We have $\Delta_{1}, \Delta_{2} \stackrel{\tau, l: L \cup L^{\prime}}{\longrightarrow} \Delta_{1}^{\prime}, \Delta_{2}^{\prime}$ with $\Delta_{1}, \Delta_{2}$ balanced. By Lemma 4.6. and [F-Com2] we have transitions $\Delta_{1}=\Delta_{3}, k: S \stackrel{k: \oplus l[L]}{\longrightarrow} \Delta_{3}, k: S^{\prime}=\Delta_{1}^{\prime}$ and $\Delta_{2}=\Delta_{4}, \bar{k}$ : $T \stackrel{\bar{k}: \& l\left[L^{\prime}\right]}{\longrightarrow} \Delta_{4}, \bar{k}: T^{\prime}=\Delta_{2}^{\prime}$ and $S \stackrel{\oplus l[L]}{\longrightarrow} S^{\prime}$ and $T \stackrel{\& l\left[L^{\prime}\right]}{\longrightarrow} T^{\prime}$. Then our original transition is on the form

$$
\Delta_{1}, \Delta_{2}=\Delta_{3}, k: S, \Delta_{4}, \bar{k}: T \stackrel{\tau, l: L \cup L^{\prime}}{\longrightarrow} \Delta_{3}, k: S^{\prime}, \Delta_{4}, \bar{k}: T^{\prime}=\Delta_{1}^{\prime}, \Delta_{2}^{\prime} .
$$

Because $\Delta_{1}, \Delta_{2}$ balanced then also $\Delta_{3}, \Delta_{4}$ is, and so $\Delta_{1}^{\prime}, \Delta_{2}^{\prime}=\Delta_{3}, k: S^{\prime}, \Delta_{4}, \bar{k}: T^{\prime}$ is balanced if $S^{\prime} \bowtie T^{\prime}$. But $S \stackrel{k: \oplus l[L]}{\longrightarrow} S^{\prime}$ implies $S=\oplus\left\{l_{j}\left[L_{j}^{\prime}\right] . S_{j}^{\prime}\right\}_{j \in J}$ with $l=l_{i}$ and $L=L_{i}$ for some $i \in J$, and $S^{\prime}=S_{i}^{\prime}$. Similarly $T \stackrel{\& l[L]}{\longrightarrow} T^{\prime}$ implies $T=\left\{l_{i}\left[L_{i}^{\prime}\right] . T_{i}^{\prime}\right\}_{i \in I}$ with $j \in I$, $l=l_{j}, L^{\prime}=L_{j}$, and $T^{\prime}=T_{j}$. Because $S \bowtie T$ we may assume $J \subseteq I$ and $i=j$, whence by definition $T_{i}^{\prime} \bowtie S_{j}^{\prime}$.

\section{Typing System for Liveness}

In this section, we introduce a variant of the standard session-typing system, give intuition for it, and establish its basic properties, notably subject reduction. In the next section, we shall prove that this typing system does indeed guarantee liveness of well-typed processes.

The central judgement will be $\Gamma ; L \vdash P \triangleright \Delta$, with the intended meaning that "with process variables $\Gamma$ and pending responses $L$, the process $P$ conforms to $\Delta$." We shall see in the next section that a well-typed lock-free $P$ is live and will eventually perform every response in $L$.

In detail, here are the environments used in the typing system, along with auxiliary operations on them.

(1) Session typing environments $\Delta$ defined at the start of Section 4.

(2) Response environments $L$ are simply sets of branch/select labels.

(3) Process variable environments $\Gamma$ are finite partial maps from process variables $X$ to tuples $(L, L, \Delta)$ or $(L, \Delta)$. We write these $(A, I, \Delta)$ for $(\mathrm{A})$ ccumulated selections and request (I)nvariant. We write $\Gamma+L$ for the environment satisfying that

$$
(\Gamma+L)(X)= \begin{cases}(A \cup L, I, \Delta) & \text { whenever } \Gamma(X)=(A, I, \Delta) \\ \Gamma(X) & \text { otherwise }\end{cases}
$$

We sometimes write $\Gamma+l$ instead of $\Gamma+\{l\}$.

Intuitively, the response environment $L$ is the set of obligations the process being typed will fulfill; every label in $L$ will eventually be selected by the process. The process variable environment assignes to each process variable two or three pieces of information. For a general recursion variable $X$, it assigns three: First an Accumulator $A$, which intuitively records the set of labels selected on a particular path through the "body" the recursion on $X$. Second, an Invariant $I$, which intuitively is the set of labels that will be selected on any path through the "body" of the recursion. Finally, $\Delta$ is the usual session environment, associating polarised channels with session types. For a primitive recursion variable $Y$, we associate simply the maximal set of responses that is allowed to be pending at the point of recursion, that is, when the process evolves to simply $Y$.

Our typing system is given in Fig. G; we proceed to explain the rules. First note that for finite processes, liveness is ensured if the process terminates with no pending responses. 


$$
\begin{aligned}
& \text { [G-OUt] } \frac{\Gamma ; L \vdash P \triangleright \Delta, k: T}{\Gamma ; L \vdash k !\langle e\rangle \cdot P \triangleright \Delta, k: ! . T} \quad \text { G-IN] } \frac{\Gamma ; L \vdash P \triangleright \Delta, k: T}{\Gamma ; L \vdash k ?(x) . P \triangleright \Delta, k: ? . T} \\
& \text { G.BRA }] \frac{\forall i \in I: \quad \Gamma+l_{i} ;\left(L \backslash l_{i}\right) \cup L_{i} \vdash P_{i} \triangleright \Delta, k: T_{i}}{\Gamma ; L \vdash k ?\left\{l_{i} \cdot P_{i}\right\}_{i \in I} \triangleright \Delta, k: \&\left\{l_{i}\left[L_{i}\right] . T_{i}\right\}_{i \in I}} \\
& \text { GR-SEL }] \frac{\Gamma+l_{j} ;\left(L \backslash l_{j}\right) \cup L_{j} \vdash P \triangleright \Delta, k: T_{j}}{\Gamma ; L \vdash k ! l_{j} . P \triangleright \Delta, k: \oplus\left\{l_{i}\left[L_{i}\right] \cdot T_{i}\right\}_{i \in I}} \quad(j \in I) \\
& \text { [G.PAR] } \frac{\Gamma ; L_{1} \vdash P_{1} \triangleright \Delta_{1} \quad \Gamma ; L_{2} \vdash P_{2} \triangleright \Delta_{2}}{\Gamma ; L_{1} \cup L_{2} \vdash P_{1} \mid P_{2} \triangleright \Delta_{1}, \Delta_{2}} \quad \text { [G.INACT] } \frac{\Delta \text { completed }}{\Gamma ; \emptyset \vdash \mathbf{0} \triangleright \Delta}
\end{aligned}
$$

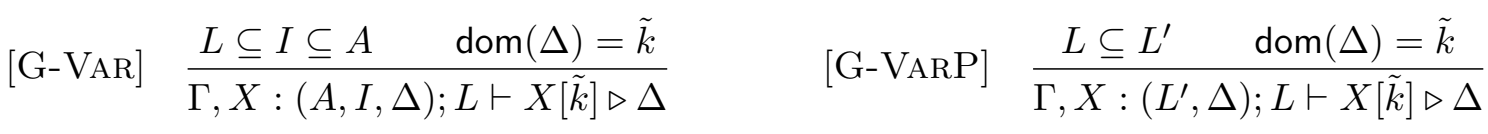

$$
\begin{aligned}
& {\left[\text { G RECP] } \frac{\Gamma, X:\left(L^{\prime}, \Delta\right) ; L^{\prime} \vdash P \triangleright \Delta \quad \Gamma ; L^{\prime} \vdash Q \triangleright \Delta \quad L \subseteq L^{\prime}}{\Gamma ; L \vdash\left(\operatorname{rec}^{e} X(i) . P ; Q\right) \triangleright \Delta}\right.} \\
& {\left[\text { G-REC] } \frac{\Gamma, X:(\emptyset, I, \Delta) ; I \vdash P \triangleright \Delta \quad L \subseteq I}{\Gamma ; L \vdash \operatorname{rec} X . P \triangleright \Delta}\right.} \\
& \text { [G-Cond }] \frac{\Gamma ; L \vdash P \triangleright \Delta \quad \Gamma ; L \vdash Q \triangleright \Delta}{\Gamma ; L \vdash \text { if } e \text { then } P \text { else } Q \triangleright \Delta}
\end{aligned}
$$

Figure G: Typing System

Hence in the rule $\mathbf{0}$, the request environment is required to be empty. For infinite processes there is no point at which we can insist on having no pending responses. Indeed, a process can be live, meeting its requirements, even though it always has some pending responses. Take for instance this process, typeable with the type used in the proof of Theorem 3.10 .

$$
\operatorname{rec} X . k ! a . k ! b . X[k] \quad \triangleright \quad k: \mu t . \oplus\left\{\begin{array}{l}
a[b]: t \\
b[a]: t
\end{array} .\right.
$$

This process has the single transition sequence

$$
P \stackrel{k \oplus a}{\longrightarrow} k ! b . P \stackrel{k \oplus b}{\longrightarrow} P \stackrel{k \oplus a}{\longrightarrow} \cdots
$$

At each state but the initial one either $b$ or $a$ is pending. Yet the process is live: any response requested in the body of the recursion is also discharged in the body, although not in order. Since infinite behaviour arises as of unfolding of recursion, responses are ensured if the body of every recursion discharges the requests of that body, even if out of order.

For general recursion, G-REC] and [G-VAR], we thus find for each recursion a set of responses, such that at most that set is requested in its body and that it responds with at least that set. In the process variable environment $\Gamma$ we record this response invariant for each variable, along with a tally of the responses performed since the start of the recursion. 
This tally is updated by the rules [G-SEL]/G-BRA] for select and branch; that is, for select, to type $k ! l . P$ wrt. $k: \oplus l\left[L^{\prime}\right] . T$, the process $P$ must do every response in $L^{\prime}$. In the hypothesis, when typing $P$, we add to this the new pending responses $L^{\prime}$. But selecting $l$ performs the response $l$, so altogether, to support pending responses $L$ in the conclusion, we must have pending responses $L \backslash\{l\} \cup L^{\prime}$ in the hypothesis. Branching is similar.

The rule for process variable [G-VAR] typing then checks that the tally includes the invariant, and that the invariant includes every currently pending response.

For primitive recursion, [G-RECP] and [G-VARP], we cannot know that the body $P$ will run at all - $e$ might evaluate to 0 . Thus, it is not helpful to tally performed responses; the type system simply checks that at the point of recursion [G-VARP], the pending responses $L$ is contained in the set of responses $L^{\prime}$ that the continuation guarantees to discharge G-RECP]. Notice that this set of $L^{\prime}$ is allowed to be weakened by the rule [G-RECP]; this is a technical necessity for weakening of $L$ to hold in general (Lemma 5.4).

Note that the syntactic restriction on the form of the body $P$ in primitive recursion (2.1) ensures that there is no issues with branching: because $P$ can contain only the process variable $X$, no branching, internal or external, can prevent the execution of the body the specified number of times.

Finally, note the use of the response environments $L$ in the rules [PAR] and [G-PAR]. For the former, because of our eventual assumption of lock-freedom, a process $P \mid Q$ will discharge the set $L$ if $P$ discharges part of $L$ and $Q$ the rest. For the latter, a conditional promising to discharge a set $L$ must obviously do so whether it takes the "true" or "false" branch.

We conclude our walk-through of the rules by showing the typing derivation of the above example process.

Example 5.1. Take $\Delta=k: \mu t .\{a[b] . t ; b[a] . t\}$. Read the derivation below bottom-up; easy to miss changes - but not all changes! - are called out with a grey box .

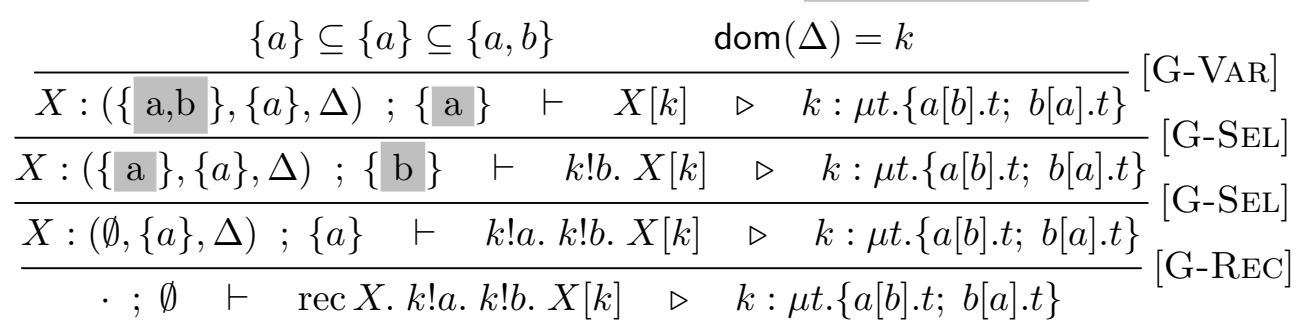

Definition 5.2. We define the standard process variable environment $\operatorname{std}(\Gamma)$ associated with a process variable environment $\Gamma$ as follows.

$$
\operatorname{std}(\Gamma)(X)=\left\{\begin{array}{cl}
\Delta & \text { whenever } \Gamma(X)=(A, I, \Delta) \\
\Delta & \text { whenever } \Gamma(X)=(I, \Delta)
\end{array}\right.
$$

Theorem 5.3. If $\Gamma ; L \vdash P \triangleright \Delta$ then also $\operatorname{std}(\Gamma) \vdash_{\text {std }} P \triangleright \Delta$.

Proof. Straightforward induction on the typing derivation, using for [G-BRA] and [G-SEL] that $\operatorname{std}(\Gamma+L)=\operatorname{std}(\Gamma)$; for [G VAR] $[\mathrm{G}-\mathrm{REC}]$ that $\operatorname{std}(\Gamma, X:(A, I, \Delta))=\operatorname{std}(\Gamma), X: \Delta$; and for $[\mathrm{G}-\mathrm{VARP}] /[\mathrm{G}-\mathrm{RECP}]$ that $\operatorname{std}(\Gamma, X:(I, \Delta))=\operatorname{std}(\Gamma), X: \Delta$.

We proceed to establish basic properties of our typing system, eventually arriving at subject reduction. First, the typing system allows weakening of the pending responses $L$.

Lemma 5.4. If $\Gamma ; L \vdash P \triangleright \Delta$ and $L^{\prime} \subseteq L$, then also $\Gamma ; L^{\prime} \vdash P \triangleright \Delta$. 
Proof. By induction on the derivation of the typing of $P$.

Case [G-InaCt]. We have $\Gamma ; L \vdash \mathbf{0} \triangleright \Delta$. By typing $L=\emptyset$ and our desired property is vacously true.

Case [G-OUT]. Immediate from the induction hypothesis.

Case [G.IN]. Immediate from the induction hypothesis.

Case [G-BRA]. We have $\Gamma ; L \vdash k ?\left\{l_{i} . P_{i}\right\} k \triangleright \Delta, k: \&\left\{l_{i}\left[L_{i}\right] . T_{i}\right\}_{i \in I}$. By typing we must have for all $i \in I$ that $\Gamma+l_{i} ;\left(L \backslash l_{i}\right) \cup L_{i} \vdash P_{i} \triangleright \Delta, k: T_{i}$. By the induction hypothesis $\Gamma+l_{i} ;\left(L^{\prime} \backslash l_{i}\right) \cup L_{i} \vdash P_{i} \triangleright \Delta, k: T_{i}$, and we conclude $\Gamma ; L^{\prime} \vdash k$ ? $\left\{l_{i} . P_{i}\right\} \triangleright \Delta, k: \&\left\{l_{i}\left[L_{i}\right] . T_{i}\right\}_{i \in I}$. Case [G-SEL]. Similar to [G-BRA].

Case [G.PAR]. We have $\Gamma ; L \vdash P_{1} \mid P_{2} \triangleright \Delta$. By typing we have $\Gamma ; L_{i} \vdash P_{i} \triangleright \Delta_{i}$ with $L=L_{1} \cup L_{2}$ and $\Delta=\Delta_{1}, \Delta_{2}$. Consider a subset $L^{\prime} \subseteq L_{1} \cup L_{2}$. By the induction hypothesis $\Gamma ; L_{i} \cap L^{\prime} \vdash P_{i} \triangleright \Delta_{i}$ and, noting that $\left(L_{1} \cap L^{\prime}\right) \cup\left(L_{2} \cap L^{\prime}\right)=\left(L_{1} \cup L_{2}\right) \cap L^{\prime}=L^{\prime}$, we find $\Gamma ; L^{\prime} \vdash P_{1} \mid P_{2} \triangleright \Delta$

Case [G VARP]. Immediate from the premise $L \subseteq L^{\prime}$.

Case [G-RECP]. Immediate from the premise $L \subseteq L^{\prime}$.

Case [G.VAR]. Immediate from the premise $L \subseteq I$.

Case [G-REC]. Immediate from the premise $L \subseteq I$.

Case [G.COND]. Immediate from the induction hypothesis.

For the subsequent development, we will need to understand how typing changes when the environment $\Gamma$ does; in particular what happens when $\Gamma(X)=(A, I, \Delta)$ and the accumulator $A$ changes. To this end, we define an ordering " $\leq$ " which captures such environments being the same except for the accumulator growing.

Definition 5.5. We define $\Gamma \leq \Gamma^{\prime}$ iff $\Gamma(X)=(A, I, \Delta)$ implies $\Gamma^{\prime}(X)=\left(A^{\prime}, I, \Delta\right)$ with $A \subseteq A^{\prime}$ and $\Gamma(X)=(I, \Delta)$ implies $\Gamma^{\prime}(X)=(I, \Delta)$.

The key insight about this ordering is then that moving from smaller to greater preserves typing, i.e., accumulators admit weakening.

Lemma 5.6. If $\Gamma ; L \vdash P \triangleright \Delta$ and $\Gamma \leq \Gamma^{\prime}$ then also $\Gamma^{\prime} ; L \vdash P \triangleright \Delta$.

Proof. Straightforward induction. We report the two essential cases.

Case [G.SEL]. We have

$$
\frac{\Gamma+l_{j} ;\left(L \backslash l_{j}\right) \cup L_{j} \vdash P \triangleright \Delta, k: T_{j}}{\Gamma ; L \vdash k ! l_{j} . P \triangleright \Delta, k: \oplus\left\{l_{i}\left[L_{i}\right] \cdot T_{i}\right\}_{i \in I}}
$$

Noting that $\Gamma \leq \Gamma^{\prime}$ implies $\Gamma+l_{j} \leq \Gamma^{\prime}+l_{j}$ we find by IH and [G-SEL]

$$
\frac{\Gamma^{\prime}+l_{j} ;\left(L \backslash l_{j}\right) \cup L_{j} \vdash P \triangleright \Delta, k: T_{j}}{\Gamma^{\prime} ; L \vdash k ! l_{j} . P \triangleright \Delta, k: \oplus\left\{l_{i}\left[L_{i}\right] . T_{i}\right\}_{i \in I}} .
$$

Case [G-REC]. We have

$$
\frac{\Gamma, X:(\emptyset, I, \Delta) ; I \vdash P \triangleright \Delta \quad L \subseteq I}{\Gamma ; L \vdash \operatorname{rec} X . P \triangleright \Delta}
$$

Noting that $\Gamma, X:(\emptyset, I, \Delta) \leq \Gamma^{\prime}, X:(\emptyset, I, \Delta)$ we have by IH and [G-REC]

$$
\frac{\Gamma^{\prime}, X:(\emptyset, I, \Delta) ; I \vdash P \triangleright \Delta \quad L \subseteq I}{\Gamma^{\prime} ; L \vdash \operatorname{rec} X . P \triangleright \Delta} .
$$


With this insight into the ordering " $\leq$ ", we can now establish that extending the environment $\Gamma$ does not change typing.

Lemma 5.7. If $\Gamma ; L \vdash P \triangleright \Delta$ then also $\Gamma+L^{\prime} ; L \vdash P \triangleright \Delta$.

Proof. Immediate from Lemma 5.6.

Lemma 5.8 (Process variable substitution). Suppose that $\Gamma, X: t ; L \vdash P \triangleright \Delta$ where either $t=\left(A, I, \Delta^{\prime}\right)$ or $t=\left(I, \Delta^{\prime}\right)$. Suppose moreover that $\Gamma ; I \vdash Q \triangleright \Delta^{\prime}$ where $X$ is not free in $Q$. Then also $\Gamma ; L \vdash P\{Q / X\} \triangleright \Delta$

Proof. By induction on the typing derivation.

Case G-Inact]. We have $\Gamma, X: t ; L \vdash \mathbf{0} \triangleright \Delta$. By typing $L=\emptyset$. Observe that $\mathbf{0}\{Q / X\}=\mathbf{0}$. Thus, by [G-InACT], we have $\Gamma ; L \vdash \mathbf{0}\{Q / X\} \triangleright \Delta$.

Case [G-OUT]. Immediate from the induction hypothesis.

Case [G-IN]. Immediate from the induction hypothesis.

Case [G-BRA]. By typing, we have

$$
\frac{\forall i \in I: \quad(\Gamma, X: t)+l_{i} ;\left(L \backslash l_{i}\right) \cup L_{i} \vdash P_{i} \triangleright \Delta, k: T_{i}}{\Gamma, X: t ; L \vdash k ?\left\{l_{i} \cdot P_{i}\right\}_{i \in I} \triangleright \Delta, k: \&\left\{l_{i}\left[L_{i}\right] . T_{i}\right\}_{i \in I}}
$$

Suppose first $t=\left(A, I, \Delta^{\prime}\right)$. Then $\left(\Gamma, X:\left(A, I, \Delta^{\prime}\right)\right)+l_{i}=\left(\Gamma+l_{i}\right), X:\left(A \cup l_{i}, I, \Delta^{\prime}\right)$. But then we may apply the induction hypothesis and [GRRA] to obtain

$$
\frac{\Gamma+l_{i} ;\left(L \backslash l_{i}\right) \cup L_{i} \vdash P_{i}\{Q / X\} \triangleright \Delta, k: T_{i}}{\Gamma ; L \vdash k ?\left\{l_{i} \cdot P_{i}\{Q / X\}\right\}_{i \in I} \triangleright \Delta, k: \&\left\{l_{i}\left[L_{i}\right] . T_{i}\right\}_{i \in I}} .
$$

Suppose instead $t=\left(I, \Delta^{\prime}\right)$. Then $\left(\Gamma, X:\left(I, \Delta^{\prime}\right)\right)+l_{i}=\left(\Gamma+l_{i}\right), X:\left(I, \Delta^{\prime}\right)$, and again we may apply the induction hypothesis and G.BRA] to obtain (5.1).

Case [G-SEL]. Similar to [G-BRA].

Case [G.PAR]. We have $\Gamma, X: t ; L \vdash P_{1} \mid P_{2} \triangleright \Delta$. By typing we find some $L_{1} \cup L_{2}=L$ and $\Delta_{1}, \Delta_{2}=\Delta$ such that $\Gamma, X: t ; L_{i} \vdash P_{i} \triangleright \Delta_{i}$. By the induction hypothesis we find $\Gamma ; L_{i} \vdash P_{i}\{Q / X\} \triangleright \Delta_{i}$, which in turn yields $\Gamma ; L_{1} \cup L_{2} \vdash\left(P_{1} \mid P_{2}\right)\{Q / X\} \triangleright \Delta_{1}, \Delta_{2}$.

Case G.VARP]. Suppose first $X \neq Y$; then by typing we have

$$
\frac{L \subseteq L^{\prime} \quad \operatorname{dom}(\Delta)=\tilde{k}}{\Gamma, Y:\left(L^{\prime}, \Delta\right), X: t ; L \vdash Y[\tilde{k}] \triangleright \Delta},
$$

so by G.VARP] also

$$
\Gamma, Y:\left(L^{\prime}, \Delta\right) ; L \vdash Y[\tilde{k}]\{Q / X\} \triangleright \Delta .
$$

If on the other hand $X=Y$ we have by typing

$$
\frac{L \subseteq L^{\prime} \quad \operatorname{dom}(\Delta)=\tilde{k}}{\Gamma, X:\left(L^{\prime}, \Delta\right) ; L \vdash X[\tilde{k}] \triangleright \Delta} ;
$$

and it must be the case that $I=L^{\prime}$ and $\Delta=\Delta^{\prime}$. We have by assumption $\Gamma ; I \vdash Q \triangleright \Delta^{\prime}$, that is $\Gamma$; $L^{\prime} \vdash Q \triangleright \Delta$. By Lemma 5.7 also $\Gamma ; L \vdash Q \triangleright \Delta$, that is, $\Gamma ; L \vdash X[\tilde{k}]\{Q / X\} \triangleright \Delta$.

Case [G-RECP]. We have $\Gamma, X:\left(A, I, \Delta^{\prime}\right) ; L \vdash\left(\operatorname{rec}^{e} Y(i) . P ; R\right) \triangleright \Delta$. By typing we have $\Gamma, X:\left(A, I, \Delta^{\prime}\right), Y:\left(L^{\prime}, \Delta\right) ; L^{\prime} \vdash P \triangleright \Delta$ and $\Gamma ; L^{\prime} \vdash R \triangleright \Delta$ for some $L^{\prime} \supseteq L$. Using $\Gamma ; I \vdash Q \triangleright \Delta^{\prime}$, by the induction hypothesis $\Gamma, Y:\left(L^{\prime}, \Delta\right) ; L^{\prime} \vdash P\{Q / X\} \triangleright \Delta$ and $\Gamma ; L^{\prime} \vdash R\{Q / X\} \triangleright \Delta$, which in turn yields $\Gamma ; L \vdash\left(\operatorname{rec}^{e} Y(i) . P ; R\right)\{Q / X\} \triangleright \Delta$. 
Case [G-VAR]. Suppose first $X \neq Y$; then by typing we have

so by [G-VAR] also

$$
\frac{L \subseteq L^{\prime} \quad \operatorname{dom}(\Delta)=\tilde{k}}{\Gamma, Y:\left(A^{\prime}, I^{\prime}, \Delta\right), X: t ; L \vdash Y[\tilde{k}] \triangleright \Delta},
$$

$$
\Gamma, Y:\left(A^{\prime}, I^{\prime}, \Delta\right) ; L \vdash Y[\tilde{k}]\{Q / X\} \triangleright \Delta .
$$

If on the other hand $X=Y$ we have by typing

$$
\frac{L \subseteq I \subseteq A \quad \operatorname{dom}(\Delta)=\tilde{k}}{\Gamma, X:\left(I, A, \Delta^{\prime}\right) ; L \vdash X[\tilde{k}] \triangleright \Delta^{\prime}} ;
$$

where necessarily $\Delta^{\prime}=\Delta$. We have by assumption $\Gamma ; I \vdash Q \triangleright \Delta^{\prime}$. By Lemma 5.7 also $\Gamma ; L \vdash Q \triangleright \Delta^{\prime}$, that is, $\Gamma ; L \vdash X[\tilde{k}]\{Q / X\} \triangleright \Delta^{\prime}$.

Case G-REC]. We have $\Gamma, X:\left(A, I, \Delta^{\prime}\right) ; L \vdash \operatorname{rec} Y . P \triangleright \Delta$. We find by typing $\Gamma, X$ : $\left(A, I, \Delta^{\prime}\right), Y:\left(A^{\prime}, I^{\prime}, \Delta\right) ; L \vdash P \triangleright \Delta$ with $L \subseteq I^{\prime}$, hence by the induction hypothesis $\Gamma, Y$ : $\left(A^{\prime}, I^{\prime}, \Delta\right) ; L \vdash P\{Q / X\} \triangleright \Delta$, and so by [G-REC] $\Gamma ; L \vdash(\operatorname{rec} Y . P)\{Q / X\} \triangleright \Delta$.

Case [G-COND]. Immediate from the induction hypothesis.

Lemma 5.9 (Term variable substitution). If $\Gamma ; L \vdash P \triangleright \Delta$ then also $\Gamma ; L \vdash P\{v / x\} \triangleright \Delta$.

Proof. Straightforward induction.

For technical reasons, we need the following two Lemmas on the presence or absence of session names in a typable process.

Lemma 5.10. If $\Gamma ; L \vdash P \triangleright \Delta, k \in \operatorname{dom}(\Delta)$, and $\Delta(k) \neq$ end then $k \in \operatorname{fn}(P)$.

Proof. Straightforward induction.

Lemma 5.11. Suppose $\Gamma ; L \vdash P \triangleright \Delta, k: T$ with $\Delta, k: T$ balanced, $T \neq$ end, and $\bar{k} \notin \mathrm{fn}(P)$. Then $\bar{k} \notin \operatorname{dom}(\Delta)$.

Proof. Supposed for a contradiction $\bar{k} \in \operatorname{dom}(\Delta)$. Because $\Delta, k: ! . T$ balanced, $\Delta(\bar{k}) \neq$ end. By Lemma 5.10 we thus have $\bar{k} \in \mathrm{fn}(P)$; contradiction.

We can now formulate the core lemma which will subsequently be used to prove subject reduction. For the formulation of the lemma, we will slightly abuse notation and consider the range of the sel(-) operator as an empty or singleton set rather than the empty or singleton sequence as which it was originally defined.

Lemma 5.12. Suppose that $\Gamma ; L \vdash P \triangleright \Delta$ with $P \stackrel{\lambda}{\rightarrow} Q$. Then there exists a type transition $\Delta \stackrel{\delta}{\rightarrow} \Delta^{\prime}$ with $\delta \simeq \lambda$, such that $\Gamma+\operatorname{sel}(\delta) ;(L \backslash \operatorname{res}(\delta)) \cup \operatorname{req}(\delta) \vdash Q \triangleright \Delta^{\prime}$. Moreover, if $\Delta$ balanced, then also $\Delta^{\prime}$ balanced.

Proof. By induction on the derivation of the transition.

Case [C-Out]. We have $k !\langle e\rangle . P \stackrel{k ! v}{\longrightarrow} P$ with $\bar{k} \notin P$ and $\Gamma ; L \vdash k !\langle e\rangle . P \triangleright \Delta, k: ! . T$. By typing $\Gamma ; L \vdash P \triangleright \Delta, k: T$. By [F-LifT] we have $k: ! . T \stackrel{k: !}{\longrightarrow} k: T$. By [F-PAR] $\Delta, k: ! . T \stackrel{k: !}{\longrightarrow} \Delta, k: T$; Observing that $k: ! \simeq k ! v$ and $\operatorname{res}(k: !)=\operatorname{sel}(k: !)=\operatorname{req}(k: !)=\emptyset$ we have found the requisite type transition.

Now suppose $\Delta, k: ! . T$ balanced; we must show $\Delta, k: T$ balanced. It is sufficient to show $\bar{k} \notin \operatorname{dom}(\Delta)$. But this follows from Lemma 5.11 . 
Case [C-In]. We have $k ?(x) . P \stackrel{k ? v}{\longrightarrow} P\{v / x\}$ with $\bar{k} \notin \mathrm{fn}(P)$ and $\Gamma ; L \vdash k ?(x) . P \triangleright \Delta, k: ? . T$. By typing $\Gamma ; L \vdash P \triangleright \Delta, k: T$. By [F-LifT] and [F-PAR], $\Delta, k: ? . T \stackrel{k: ?}{\longrightarrow} \Delta, k: T$. By Lemma 5.9 we have $\Gamma ; L \vdash P\{v / x\} \triangleright \Delta, k: T$. Observing that req $\left(k: ? T\left[L^{\prime}\right]\right)=\operatorname{sel}\left(k: ? T\left[L^{\prime}\right]\right)=$ $\operatorname{res}\left(k: ? T\left[L^{\prime}\right]\right)=\emptyset$ and that $k: ? \simeq k ? v$ we have found the requisite transition and typing. Preservation of balance follows from Lemma 5.11 .

Case [C-BRA]. We have $k ?\left\{l_{i} . P_{i}\right\} \stackrel{k \& l_{i}}{\longrightarrow} P_{i}$ and $\Gamma ; L \vdash k ?\left\{l_{i} . P_{i}\right\}_{i \in I} \triangleright \Delta, k: \&\left\{l_{i}\left[L_{i}\right] . T_{i}\right\}_{i \in I}$. By typing we have $\Gamma+l_{i} ;\left(L \backslash\left\{l_{i}\right\}\right) \cup L_{i} \vdash P_{i} \triangleright \Delta, k: T_{i}$. By [F-LifT] and [F-PAR] we have $\Delta, k: \&\left\{l_{i}\left[L_{i}\right] \cdot T_{i}\right\}_{i \in I} \stackrel{k: \& l_{i}\left[L_{i}\right]}{\longrightarrow} \Delta, k: T_{i}$. Observing that req $\left(k: \& l_{i}\left[L_{i}\right]\right)=L_{i}$, $\operatorname{sel}\left(k: \& l_{i}\left[L_{i}\right]\right)=\operatorname{res}\left(k: \& l_{i}\left[L_{i}\right]\right)=\left\{l_{i}\right\}$ and that $k: \oplus l_{i}\left[L_{i}\right] \simeq k \& l_{i}$, we have found the requisite type transition. Preservation of balance follows from Lemma 5.11 .

Case [C-SEL]. We have $k ! l . P \stackrel{k \oplus l_{i}}{\longrightarrow} P$ and $\Gamma ; L \vdash k ! l_{i} . P \triangleright \Delta, k: \oplus\left\{l_{i}\left[L_{i}\right] . T_{i}\right\}_{i \in I}$. By typing $\Gamma+l_{i} ;\left(L \backslash\left\{l_{i}\right\}\right) \cup L_{i} \vdash P \triangleright \Delta, k: T_{i}$. By [F-LifT] and [F-PAR] we have

$$
\Delta, \oplus\left\{l_{i}\left[L_{i}\right] . T_{i}\right\}_{i \in I} \stackrel{k: \oplus l_{i}\left[L_{i}\right]}{\longrightarrow} \Delta, T_{i} .
$$

Observing that $\operatorname{req}\left(k: \oplus l_{i}\left[L_{i}\right]\right)=L_{i}$, sel $\left(k: \oplus l_{i}\left[L_{i}\right]\right)=\operatorname{res}\left(k: \oplus l_{i}\left[L_{i}\right]\right)=\left\{l_{i}\right\}$ and that $k: \oplus l_{i}\left[L_{i}\right] \simeq k \oplus l_{i}$, we have found the requisite type transition. Preservation of balance follows from Lemma 5.11 .

Case [C.PARL]. We have $P\left|P^{\prime} \stackrel{\lambda}{\rightarrow} Q\right| P^{\prime}$ with $\overline{\operatorname{subj}(\lambda)} \notin \mathrm{fn}\left(P^{\prime}\right)$ and $\Gamma ; L \vdash P \mid P^{\prime} \triangleright \Delta$. By typing we have for some $L_{1} \cup L_{2}=L$ and $\Delta_{1} \cup \Delta_{2}$ that $\Gamma ; L_{1} \vdash P \triangleright \Delta_{1}$ and $\Gamma ; L_{2} \vdash P^{\prime} \triangleright \Delta_{2}$. By the induction hypothesis, we have a transition $\Delta_{1} \stackrel{\delta}{\rightarrow} \Delta_{1}^{\prime}$ with $\Gamma+\operatorname{sel}(\delta) ; L_{1} \backslash \operatorname{res}(\delta) \cup$ $\operatorname{req}(\delta) \vdash Q \triangleright \Delta_{1}^{\prime}$ and $\delta \simeq \lambda$. By Lemma 5.7 we find also $\Gamma+\operatorname{sel}(\delta) ; L_{2} \vdash P^{\prime} \triangleright \Delta_{2}$. By Lemma $4.5 \operatorname{dom}\left(\Delta_{1}\right)=\operatorname{dom}\left(\Delta_{1}^{\prime}\right)$ so $\Delta_{1}^{\prime}, \Delta_{2}$ is defined, and hence by [G-PAR] we have $\Gamma+\operatorname{sel}(\delta) ; L_{1} \backslash \operatorname{res}(\delta) \cup \operatorname{req}(\delta) \cup L_{2} \vdash Q \mid P^{\prime} \triangleright \Delta_{1}^{\prime}, \Delta_{2}$. This is not exactly the form we need, but observing that

$$
\left(L_{1} \cup L_{2}\right) \backslash \operatorname{res}(\delta) \cup \operatorname{req}(\delta) \subseteq\left(L_{1} \backslash \operatorname{res}(\delta)\right) \cup \operatorname{req}(\delta) \cup L_{2},
$$

we find again by Lemma 5.4 that $\Gamma+\operatorname{sel}(\delta) ;\left(L_{1} \cup L_{2}\right) \backslash \operatorname{res}(\delta) \cup \operatorname{req}(\delta) \vdash Q \mid P^{\prime} \triangleright \Delta_{1}^{\prime}, \Delta_{2}$. By [F-PAR] $\Delta_{1}, \Delta_{2} \stackrel{\delta}{\rightarrow} \Delta_{1}^{\prime}, \Delta_{2}$, and we have found the requisite type transition.

Now suppose $\Delta_{1}, \Delta_{2}$ balanced. By Lemma 4.7 it is sufficient to prove that $\overline{\operatorname{subj}(\delta)} \notin$ $\operatorname{dom}\left(\Delta_{1}, \Delta_{2}\right)$. If $\operatorname{subj}(\delta)=\tau$ this is trivial, so say $\operatorname{subj}(\delta)=k$ and suppose for a contradiction $\bar{k} \in \operatorname{dom}\left(\Delta_{1}, \Delta_{2}\right)$. We must have $\delta=k: \rho$ and because $\delta \simeq \lambda$ we must have $\operatorname{subj}(\lambda)=$ $\operatorname{subj}(\delta)=k$. By Lemma $2.2 \bar{k} \notin \mathrm{fn}\left(Q \mid P^{\prime}\right)$. By Lemma 4.6 we have $\Delta_{1}=\Delta_{1}^{\prime \prime}, k: S$ with $S \neq$ end. Because $\Delta_{1}, \Delta_{2}$ balanced, $\left(\Delta_{1}, \Delta_{2}\right)(\bar{k}) \bowtie S$ and so $\left(\Delta_{1}, \Delta_{2}\right)(\bar{k}) \neq$ end.

Suppose first $\bar{k} \in \operatorname{dom}\left(\Delta_{1}\right)$. Then $\bar{k} \in \operatorname{dom}\left(\Delta_{1}^{\prime \prime}\right)$, so also $\Delta^{\prime \prime}(\bar{k}) \neq$ end, and it follows that $\Delta_{1}^{\prime}(\bar{k})=\Delta_{1}^{\prime \prime}(\bar{k}) \neq$ end. By Lemma $5.10 \bar{k} \in \mathrm{fn}(Q)$, contradicting $\bar{k} \notin \mathrm{fn}\left(Q \mid P^{\prime}\right)$.

Suppose instead $\bar{k} \in \operatorname{dom}\left(\Delta_{2}\right)$. Then immediately by Lemma $5.10 \bar{k} \in \mathrm{fn}\left(P^{\prime}\right)$, contradicting $\bar{k} \notin \mathrm{fn}\left(Q \mid P^{\prime}\right)$.

Case [C-Com1]. We have

$$
\frac{P_{1} \stackrel{\bar{k} ! v}{\rightarrow} P_{1}^{\prime} \quad P_{2} \stackrel{k ? v}{\rightarrow} P_{2}^{\prime}}{P_{1}\left|P_{2} \stackrel{\tau}{\rightarrow} P_{1}^{\prime}\right| P_{2}^{\prime}}
$$

and

$$
\frac{\Gamma ; L_{1} \vdash P_{1} \triangleright \Delta_{1} \quad \Gamma ; L_{2} \vdash P_{2} \triangleright \Delta_{2}}{\Gamma ; L_{1} \cup L_{2} \vdash P_{1} \mid P_{2} \triangleright \Delta_{1}, \Delta_{2}}
$$


By the induction hypothesis we find $\Delta_{i} \stackrel{\delta_{i}}{\rightarrow} \Delta_{i}^{\prime}$ s.t. $\Gamma+\operatorname{sel}\left(\delta_{i}\right) ; L_{i} \backslash \operatorname{res}\left(\delta_{i}\right) \cup \operatorname{req}\left(\delta_{i}\right) \vdash P_{i} \triangleright \Delta_{i}^{\prime}$ with $\delta_{1} \simeq \bar{k} ! v$ and $\delta_{2} \simeq k ? v$. It follows that $\delta_{1}=k: !$ and $\delta_{2}=k: ?$ whence res $\left(\delta_{1}\right)=\operatorname{req}\left(\delta_{2}\right)=$ $\operatorname{res}\left(\delta_{2}\right)=\operatorname{req}\left(\delta_{1}\right)=\emptyset$ and $\operatorname{sel}\left(\delta_{1}\right)=\operatorname{sel}\left(\delta_{2}\right)=\epsilon$. By Lemma $4.5 \Delta_{1}^{\prime}, \Delta_{2}^{\prime}$ defined, and so by [F-Com1] we have $\Delta_{1}, \Delta_{2} \stackrel{\tau}{\rightarrow} \Delta_{1}^{\prime}, \Delta_{2}^{\prime}$. Noting that $\tau \simeq \tau$ and that $\Gamma+\operatorname{sel}\left(\delta_{1}\right)+\operatorname{sel}\left(\delta_{2}\right)=\Gamma$, we have the required type transition. $\operatorname{Since} \operatorname{subj}(\tau)=\tau$ and $\operatorname{so} \overline{\operatorname{subj}(\tau)} \notin \operatorname{dom}\left(\Delta_{1}, \Delta_{2}\right)$, it follows from Lemma 4.7 that $\Delta_{1}^{\prime}, \Delta_{2}^{\prime}$ is balanced when $\Delta_{1}, \Delta_{2}$ is.

Case [C-Com2]. We have

$$
\frac{P_{1} \stackrel{\bar{k} \oplus l}{\longrightarrow} P_{1}^{\prime} \quad P_{2} \stackrel{k \& l}{\longrightarrow} P_{2}^{\prime}}{P_{1}\left|P_{2} \stackrel{\tau: l}{\longrightarrow} P_{1}^{\prime}\right| P_{2}^{\prime}}
$$

and

$$
\frac{\Gamma ; L_{1} \vdash P_{1} \triangleright \Delta_{1} \quad \Gamma ; L_{2} \vdash P_{2} \triangleright \Delta_{2}}{\Gamma ; L_{1} \cup L_{2} \vdash P_{1} \mid P_{2} \triangleright \Delta_{1}, \Delta_{2}}
$$

By induction we find $\Delta_{i} \stackrel{\delta_{i}}{\rightarrow} \Delta_{i}^{\prime}$ s.t. $\Gamma+\operatorname{sel}\left(\delta_{i}\right) ; L_{i} \backslash \operatorname{res}\left(\delta_{i}\right) \cup \operatorname{req}\left(\delta_{i}\right) \vdash P_{i} \triangleright \Delta_{i}^{\prime}$ with $\delta_{1} \simeq \bar{k} \oplus l$ and $\delta_{2} \simeq k \& l$. It follows that for some $L_{1}^{\prime}, L_{2}^{\prime}$ we have $\delta_{1}=\bar{k}: \oplus l\left[L_{1}^{\prime}\right]$ and $\delta_{2}=k: \& l\left[L_{2}^{\prime}\right]$, and so req $\left(\delta_{1}\right)=L_{1}^{\prime}$ and $\operatorname{req}\left(\delta_{2}\right)=L_{2}^{\prime}, \operatorname{res}\left(\delta_{1}\right)=\operatorname{res}\left(\delta_{2}\right)=\{l\}$, and $\operatorname{sel}\left(\delta_{1}\right)=\operatorname{sel}\left(\delta_{2}\right)=l$. By Lemma $4.5 \Delta_{1}^{\prime}, \Delta_{2}^{\prime}$ defined, and so we find a transition $\Delta_{1}, \Delta_{2} \stackrel{\tau: l, L_{1}^{\prime} \cup L_{2}^{\prime}}{\longrightarrow} \Delta_{1}^{\prime}, \Delta_{2}^{\prime}$ by [F-Com2]. By [GF-PAR] we find $\Gamma+l ; L_{1} \backslash\{l\} \cup L_{1}^{\prime} \cup L_{2} \backslash\{l\} \cup L_{2}^{\prime} \vdash P_{1}^{\prime} \mid P_{2}^{\prime} \triangleright \Delta_{1}^{\prime}, \Delta_{2}^{\prime}$. Noting that $\tau: l, L_{1}^{\prime} \cup L_{2}^{\prime} \simeq \tau: l$ and

$$
\begin{aligned}
L_{1} \backslash\{l\} \cup L_{1}^{\prime} \cup L_{2} \backslash\{l\} \cup L_{2}^{\prime} & =\left(L_{1} \cup L_{2}\right) \backslash\{l\} \cup L_{1}^{\prime} \cup L_{2}^{\prime} \\
& =\left(L_{1} \cup L_{2}\right) \backslash \operatorname{res}\left(\tau: l, L_{1}^{\prime} \cup L_{2}^{\prime}\right) \cup \operatorname{req}\left(\tau: l, L_{1}^{\prime} \cup L_{2}^{\prime}\right),
\end{aligned}
$$

we have the required type transition. $\operatorname{Since} \operatorname{subj}\left(\tau: l, L_{1}^{\prime} \cup L_{2}^{\prime}\right)=\tau$ and $\operatorname{so} \overline{\operatorname{subj}\left(\tau: l, L_{1}^{\prime} \cup L_{2}^{\prime}\right)} \notin$ $\operatorname{dom}\left(\Delta_{1}, \Delta_{2}\right)$, it follows from Lemma 4.7 that $\Delta_{1}^{\prime}, \Delta_{2}^{\prime}$ is balanced when $\Delta_{1}, \Delta_{2}$ is.

Case [C-REC]. We have

$$
\frac{P\{\operatorname{rec} X . P / X\} \stackrel{\lambda}{\rightarrow} Q}{\operatorname{rec} X . P \stackrel{\lambda}{\rightarrow} Q}
$$

and

$$
\frac{\Gamma, X:(\emptyset, I, \Delta) ; I \vdash P \triangleright \Delta \quad L \subseteq I}{\Gamma ; L \vdash \operatorname{rec} X . P \triangleright \Delta}
$$

It follows by [G-REC] that also $\Gamma ; I \vdash \operatorname{rec} X . P \triangleright \Delta$ and by Lemma 5.7 that $\Gamma, X:(\emptyset, I, \Delta) ; L \vdash$ $P \triangleright \Delta$. It then follows by Lemma 5.8 that

$$
\Gamma ; L \vdash P\{\operatorname{rec} X . P / X\} \triangleright \Delta .
$$

By the induction hypothesis we find a balance-preserving type transition $\Delta \stackrel{\delta}{\rightarrow} \Delta^{\prime}$ with $\delta \simeq \lambda$ and $\Gamma+\operatorname{sel}(\delta) ; L \backslash \operatorname{res}(\delta) \cup \operatorname{req}(\delta) \vdash Q \triangleright \Delta^{\prime}$.

Case [C-Prec0]. We have

$$
\frac{e \Downarrow 0 \quad Q \stackrel{\lambda}{\rightarrow} R}{\left(\operatorname{rec}^{e} X(i) . P ; Q\right) \stackrel{\lambda}{\rightarrow} R}
$$

and

$$
\frac{\Gamma, X:\left(L^{\prime}, \Delta\right) ; L^{\prime} \vdash P \triangleright \Delta \quad \Gamma ; L^{\prime} \vdash Q \triangleright \Delta \quad L \subseteq L^{\prime}}{\Gamma ; L \vdash\left(\operatorname{rec}^{e} X(i) . P ; Q\right) \triangleright \Delta} .
$$

By Lemma 5.7 we have also $\Gamma ; L \vdash Q \triangleright \Delta$, and so by the induction hypothesis we find the required balance-preserving type transition. 
Case [C-PreCN]. We have

$$
\frac{e \Downarrow n+1 \quad P\{n / i\}\left\{\left(\operatorname{rec}^{n} X(i) \cdot P ; Q\right) / X\right\} \stackrel{\lambda}{\rightarrow} R}{\left(\operatorname{rec}^{e} X(i) \cdot P ; Q\right) \stackrel{\lambda}{\rightarrow} R}
$$

and again

$$
\frac{\Gamma, X:\left(L^{\prime}, \Delta\right) ; L^{\prime} \vdash P \triangleright \Delta \quad \Gamma ; L^{\prime} \vdash Q \triangleright \Delta \quad L \subseteq L^{\prime}}{\Gamma ; L \vdash\left(\operatorname{rec}^{e} X(i) . P ; Q\right) \triangleright \Delta} .
$$

By [GRECP] it follows that $\Gamma ; L^{\prime} \vdash\left(\operatorname{rec}^{n} X(i) . P ; Q\right) \triangleright \Delta$. By Lemmas 5.9 and 5.4 we have $\Gamma, X:\left(L^{\prime}, \Delta\right) ; L \vdash P\{n / i\} \triangleright \Delta$. Finally, by Lemma 5.8 we have

$$
\Gamma ; L \vdash P\{n / i\}\left\{\left(\operatorname{rec}^{n} X(i) . P ; Q\right) / X\right\} \triangleright \Delta,
$$

and the requisite balance-preserving type transition follows by the induction hypothesis.

Case [C-CondT] and [C-CondF]. We have,

$$
\frac{e \Downarrow \text { true } \quad P \stackrel{\lambda}{\rightarrow} P^{\prime}}{\text { if } e \text { then } P \text { else } Q \stackrel{\lambda}{\rightarrow} P^{\prime}}
$$

and

$$
\frac{\Gamma ; L \vdash P \triangleright \Delta \quad \Gamma ; L \vdash Q \triangleright \Delta}{\Gamma ; L \vdash \text { if } e \text { then } P \text { else } Q \triangleright \Delta},
$$

and the requisite balance-preserving type transition follows from the induction hypothesis. The other case is the same.

Theorem 5.13 (Subject reduction). Suppose that $\cdot ; L \vdash P \triangleright \Delta$ and $P \stackrel{\lambda}{\rightarrow} Q$. Then there exists a type transition $\Delta \stackrel{\delta}{\rightarrow} \Delta^{\prime}$ with $\delta \simeq \lambda$, such that $\cdot ;(L \backslash \operatorname{res}(\delta)) \cup \operatorname{req}(\delta) \vdash Q \triangleright \Delta^{\prime}$. Moreover, if $\Delta$ balanced then also $\Delta^{\prime}$ balanced.

Proof. Immediate from the Lemma 5.12 .

Example 5.14. With the system of Figure G, the process $P(D)$ is typable wrt. the types given in Example 3.4. The process $P\left(D_{0}\right)$ on the other hand is not: We have $\cdot \emptyset \vdash P(D) \triangleright k: T_{P}, o^{+}: T_{D}, o^{-}: \overline{T_{D}}$, but the same does not hold for $P\left(D_{0}\right)$. We also exemplify a typing judgment with non-trivial guaranteed responses. The process $D$, the order-fulfillment part of $P(D)$, can in fact be typed

$$
\cdot ; \text { SI }\} \vdash D \triangleright k: \mu t^{\prime} . \oplus\left\{\text { DI.!.t } t^{\prime} \text { SI.!.end }\right\}, o^{-}: \overline{T_{D}}
$$

Note the left-most $\{\mathrm{SI}\}$, indicating intuitively that this process will eventually select $\mathrm{SI}$ in any execution. The process $D$ has this property essentially because it is implemented by bounded recursion.

\section{LIVENESS}

We now prove that if a lock-free process is well-typed under our liveness typing system, then that process is indeed live.

However, we will need a bit of a detour to define lock-free processes: Lock-freedom is defined in terms of maximal transition sequences, which are in turn defined using (weak) fairness. For both lock-freedom and fairness, we will in turn need to speak about occurrences of the communication actions $k !\langle e\rangle, k ?(x), k ! l$., and $k ?\{-\}$.

The roadmap for this Section thus becomes: 
(1) In Sub-section 6.1, we identify key properties of occurrences; then use these properties to define fairness, maximal transition sequences, and lock-freedom; and finally, what it means for a process to be live.

(2) In Sub-section 6.2 We establish decomposition properties of maximal transition sequences for parallel compositions, especially wrt. the responses performed by such processes.

(3) We build on these properties and the syntactic restrictions on primtive recursion when in Sub-section 6.3, we establish technical properties of primitive recursion.

(4) Finally, can prove in Sub-section 6.4 first that well-typed open lock-free processes are constrained in what responses they might require by the response invariant of its process variables on the one hand, and the responses it will always do in the other. This is enough to prove that well-typed closed lock-free processes are necessarily live.

6.1. Occurrences and liveness. Since we do not employ a structural congruence, defining occurrences and liveness is straightforward, and we simply assume we can identify occurrences across transitions through residuals - a rigorous treatment is in [3]; see also [17]. Given an occurrence of an action $a$ in $P$, we may thus speak of that occurrence being preserved by a transition $P \stackrel{\lambda}{\rightarrow} Q$ if it remains after the transition, or being executed if it is consumed by the transition. Similarly, we will say that an occurrence of an action is enabled if it is executed by some transition, and top-level if it is not nested inside another action.

We will rely on the following properties of occurrences and transitions.

Lemma 6.1. Occurrences have the following properties.

(1) If an occurrence is enabled, it is also top-level.

(2) If $P \stackrel{\lambda}{\rightarrow} Q$ preserves a top-level occurrence of an action a in $P$, then that occurrence is also top-level in $Q$.

(3) If $P \stackrel{\lambda}{\rightarrow} Q$ then there exists an occurrence of an action a in $P$ which is executed by that transition.

Using occurrences, we define fairness following [17].

Definition 6.2. An infinite transition sequence $s=\left(P_{i}, \lambda_{i}\right)_{i \in \mathbb{N}}$ is fair iff whenever an occurrence of an action $a$ occurs enabled in $P_{n}$ then some $m \geq n$ has $P_{m} \stackrel{\lambda_{m}}{\longrightarrow} P_{m+1}$ executing that occurrence.

Example 6.3. Consider the process $P=(\operatorname{rec} X . k ! a . X[k]) \mid(\operatorname{rec} Y . h ! a . Y[h])$. This process has an infinite execution

$$
P \stackrel{k ! a}{\longrightarrow} P \stackrel{k ! a}{\longrightarrow} \cdots
$$

however, this execution is not fair, since the occurrence of $h ! a$ is enabled in the initial process $P$, but never executed. Conversely, the infinite execution

$$
P \stackrel{k ! a}{\longrightarrow} P \stackrel{h ! a}{\longrightarrow} P \stackrel{k ! a}{\longrightarrow} \cdots
$$

(note the second label $h ! a$ ) is fair, since, indexing the (identical) processes $P$ by $P=P_{1}, \ldots$, for any $i$, the enabled occurrences of actions in $P_{i}$ are $k ! a$ and $h ! a$, and these are executed at either $P_{i+1}$ or $P_{i+2}$.

Definition 6.4. A transition sequence $s$ is terminated iff it has finite length $n$ and $P_{n} \nrightarrow$. It is maximal iff it is finite and terminated or infinite and fair. 
The transition sequence (6.1) above is maximal.

We define lock-freedom in the spirit of [24]; notice that the present definition strictly generalises fairness.

Definition 6.5. A maximal transition sequence $\left(P_{i}, \lambda_{i}\right)$ is lock-free iff whenever there is a top-level occurrence of an action $a$ in $P_{i}$, then there exists some $j \geq i$ s.t. $P_{j} \stackrel{\lambda_{j}}{\longrightarrow} P_{j+1}$ executes that occurrence. A process is lock-free iff all its transition sequences are.

This definition adapts the one of [24] to the setting of binary session types. However, the present definition is given in terms of the transition semantics as opposed to the reduction semantics. Because of our amended [S-PARL] rule, this makes sense: a process has a non- $\tau$ transition $\lambda$ precisely if it holds only one half of a session, and thus needs and expects an environment to perform a co-action in order to proceed. However, since our definition of lock-freedom imposes its condition on every maximal transition sequence, there is no presumption of a cooperative environment.

Example 6.6. The infinite execution sequence (6.1) above is lock-free. Conversely, consider this process.

$$
Q=(\operatorname{rec} X . k ! a . X[k])|h ?(x) \cdot \bar{j} !\langle x\rangle| j ?(y) \cdot \bar{h} !\langle y\rangle
$$

Because [SPAR-L] does not permit transitions on neither $h$ nor $j, Q$ has only one infinite transition sequence:

$$
Q \stackrel{k ! a}{\longrightarrow} Q \stackrel{k ! a}{\longrightarrow} \cdots .
$$

This sequence is maximal but not lock-free. Again indexing the processing in the sequence $Q=Q_{1}, Q_{2}, \ldots$ we see that at each $Q_{i}$ we have only one enabled occurrence of an action, namely $k ! a$, which is immediately consumed by a transition. Hence this sequence is maximal. On the other hand, at each $Q_{i}$ we have top-level occurrences of both $h ? x$ and $j ? y$, but neither is ever executed. Hence this sequence is not lock-free.

Using the notion of maximal transition sequence, we can now say what it means for a process to be live.

Definition 6.7 (Live process). A well-typed process $\Theta \vdash_{\text {std }} P \triangleright \Delta$ is live wrt. $\Theta, \Delta$ iff for any maximal transition sequence $\left(P_{i}, \lambda_{i}\right)_{i}$ of $P$ there exists a live typed transition sequence $\left(\Delta_{i}, \delta_{i}\right)_{i}$ of $\Delta$ s.t. $\left(\left(P_{i}, \Delta_{i}\right),\left(\lambda_{i}, \delta_{i}\right)\right)_{i}$ is a typed transition sequence of $\Theta \vdash_{\text {std }} P \triangleright \Delta$.

6.2. Decomposition of transition sequences. We proceed to establish properties of transition sequences of a parallel process: Most importantly, they arise as the merge of transition sequences of their underlying left and right processes. First, an auxiliary definition.

Definition 6.8. For a process transition label $\lambda$, define $\operatorname{sel}(\lambda)$ by

$$
\begin{gathered}
\operatorname{sel}(k ! v)=\operatorname{sel}(k ? v)=\operatorname{sel}(\tau)=\emptyset \\
\operatorname{sel}(k \& l)=\operatorname{sel}(k \oplus l)=\operatorname{sel}(\tau: l)=l
\end{gathered}
$$

Given a trace $\alpha$ we lift sel $(-)$ pointwise, that is, $\operatorname{sel}(\alpha)=\left\{\operatorname{sel}(\lambda) \mid \alpha=\phi \lambda \alpha^{\prime}\right\}$.

Note that $\delta \simeq \lambda$ implies $\operatorname{sel}(\lambda)=\operatorname{sel}(\delta)$.

Lemma 6.9. For any transition sequence $s$ of $P \mid Q$, there exists transition sequences $p=\left(P_{i}, \beta_{i}\right)_{i \in|p|}$ and $q=\left(Q_{i}, \delta_{i}\right)_{i \in|q|}$ and monotone surjective maps $u:|s| \rightarrow|p|$ and $v:|s| \rightarrow|q|$ such that $s=\left(\left(P_{u(i)} \mid Q_{v(i)}\right), \alpha_{i}\right)_{i \in|s|}$ and $\operatorname{sel}(\beta) \cup \operatorname{sel}(\delta)=\operatorname{sel}(\alpha)$. 
Proof. We prove the existence of such functions for finite $s$; the result for infinite $s$ follows. So suppose $s$ is finite and write it $s=\left(S_{i}, \alpha_{i}\right)_{i \in|s|}$. We proceed by induction on the length of $s$. First, a bit of notation: when $\alpha=\alpha_{1} \ldots \alpha_{n}$ we define cut $(\alpha)=\alpha_{1} \ldots \alpha_{n-1}$. Now, for $|s|=1$, the identity functions suffice. Suppose instead $|s|=n+1$, and consider the last transition $S_{n} \stackrel{\alpha_{n}}{\longrightarrow} S_{n+1}$. By the induction hypothesis we have transition sequences $p, q$ with labels $\beta, \delta$ and maps $u, v$ such that $S_{n}=P_{u(n)} \mid Q_{v(n)}$ and $\operatorname{sel}(\beta) \cup \operatorname{sel}(\delta)=\operatorname{sel}(\operatorname{cut}(\alpha))$ etc. Notice that because $u, v$ are surjective and monotone, $p, q$ must have lengths $u(n)$ and $v(n)$, respectively. We proceed by cases on the derivation of this last transition.

Case [C-PARL]. We must in this case have

$$
\frac{P_{u(n)} \stackrel{\alpha_{n}}{\longrightarrow} R}{P_{u(n)}\left|Q_{v(n)} \stackrel{\alpha_{n}}{\longrightarrow} R\right| Q_{v(n)}=S_{n+1}} .
$$

Extend $p$ to $p^{\prime}$ by taking $P_{u(n)+1}=R$ and $\beta_{u(n)}=\alpha_{n}$; and extend $u, v$ by taking $u(n+1)=$ $u(n)+1$ and $v(n+1)=v(n)$ and we have found the requisite transition sequences and maps. It is now sufficient to note that

$$
\begin{aligned}
\operatorname{sel}(\alpha) & =\operatorname{sel}\left(\alpha_{n}\right) \cup \operatorname{sel}(\operatorname{cut}(\alpha)) \\
& =\operatorname{sel}\left(\beta_{u(n)}\right) \cup \operatorname{sel}(\beta) \cup \operatorname{sel}(\delta) \\
& =\operatorname{sel}\left(\beta^{\prime}\right) \cup \operatorname{sel}(\delta) .
\end{aligned}
$$

Case [C-Com1]. We must have in this case

$$
\frac{P_{u(n)} \stackrel{\bar{k} ! v}{\longrightarrow} P^{\prime} \quad Q_{v(n)} \stackrel{k ? v}{\longrightarrow} Q^{\prime}}{P_{u(n)}\left|Q_{v(n)} \stackrel{\tau=\alpha_{n}}{\longrightarrow} P^{\prime}\right| Q^{\prime}=S_{n+1}} .
$$

Extend $p$ to $p^{\prime}$ by taking $P_{u(n)+1}=P^{\prime}$ and $\beta_{u(n)}=\bar{k} ! v$; and similarly extend $q$ to $q^{\prime}$ by taking $Q_{v(n)+1}=Q^{\prime}$ and $\delta_{v(n)}=k ? v$. Extending also $u, v$ by $u(n+1)=u(n)+1$ and $v(n+1)=v(n)+1$ we have found the requisite transition sequences and maps. It is now sufficient to note that

$$
\begin{aligned}
\operatorname{sel}(\alpha) & =\operatorname{sel}(\tau) \cup \operatorname{sel}(\operatorname{cut}(\alpha)) \\
& =\operatorname{sel}(\beta) \cup \operatorname{sel}(\delta) \\
& =\operatorname{sel}(\bar{k} ! v) \cup \operatorname{sel}\left(\beta^{\prime}\right) \cup \operatorname{sel}(k ? v) \cup \operatorname{sel}\left(\delta^{\prime}\right) \\
& =\operatorname{sel}\left(\beta^{\prime}\right) \cup \operatorname{sel}\left(\delta^{\prime}\right)
\end{aligned}
$$

Case [C-Com2]. We must have in this case

$$
\frac{P_{u(n)} \stackrel{\bar{k} \oplus l}{\longrightarrow} P^{\prime} \quad Q_{v(n)} \stackrel{k \& l}{\longrightarrow} Q^{\prime}}{P_{u(n)}\left|Q_{v(n)} \stackrel{\tau: l=\alpha_{n}}{\longrightarrow} P^{\prime}\right| Q^{\prime}=S_{n+1}} .
$$

Extend $p$ to $p^{\prime}$ by taking $P_{u(n)+1}=P^{\prime}$ and $\beta_{u(n)}=\bar{k} \oplus l$; and similarly extend $q$ to $q^{\prime}$ by taking $Q_{v(n)+1}=Q^{\prime}$ and $\delta_{v(n)}=k \& l$. Extending also $u, v$ by $u(n+1)=u(n)+1$ and $v(n+1)=v(n)+1$ we have found the requisite transition sequences and maps. It is now 
sufficient to note that

$$
\begin{aligned}
\operatorname{sel}(\alpha) & =\operatorname{sel}(\tau: l) \cup \operatorname{sel}(\operatorname{cut}(\alpha)) \\
& =\{l\} \cup \operatorname{sel}(\beta) \cup \operatorname{sel}(\delta) \\
& =\operatorname{sel}(\bar{k} \oplus l) \cup \operatorname{sel}(\beta) \cup \operatorname{sel}(k \& l) \cup \operatorname{sel}(\delta) \\
& =\operatorname{sel}\left(\beta^{\prime}\right) \cup \operatorname{sel}\left(\delta^{\prime}\right)
\end{aligned}
$$

Lemma 6.10. If $s$ is a maximal lock-free transition sequence of $P \mid Q$ with trace $\alpha$, then there exist maximal lock-free transition sequences $p, q$ of $P, Q$ with traces $\beta, \delta$, respectively, such that $\operatorname{sel}(\alpha)=\operatorname{sel}(\beta) \cup \operatorname{res}(\delta)$.

Proof. By Lemma 6.9 we find transition sequences $p=\left(P_{i}, \beta_{i}\right)_{i \in|p|}$ and $q=\left(Q_{i}, \delta_{i}\right)_{i \in|q|}$ and maps $u, v$ such that $s$ can be written $s=\left(s_{i}, \alpha_{i}\right)_{i \in|s|}=\left(P_{u(i)} \mid Q_{v(i)}, \alpha_{i}\right)_{i \in|s|}$ and $\operatorname{sel}(\alpha)=\operatorname{sel}(\beta) \cup \operatorname{sel}(\delta)$. It remains to prove that these $p, q$ are maximal and lock-free. Suppose for a contradiction that $p$ is not; the case for $q$ is similar. Then either (A) $p$ is maximal but not lock-free, or (B) $p$ is not maximal.

We consider first (A); $p$ maximal but not lock-free. Then some top-level occurrence of an action $a$ sits in each $P_{i}$ when $i \geq n$ for some $n$. But then for $j \geq u^{-1}(n)$ we must have $s_{j}=\left(P_{u(n)}, Q_{v(n)}\right)$ contradicting $s$ lock-free.

Consider now (B); $p$ not maximal. Then either (1) $p$ is finite and can be extended by a transition $\lambda$, or (2) $p$ is infinite but not fair.

Suppose (1) that is, $p$ of finite length $n$ and $P_{n} \stackrel{\lambda}{\rightarrow}$. By Lemma 6.1 (3) $P_{n}$ must have an occurrence of an enabled action $a$. By Lemma 6.1(1) this occurrence is top-level. But for $i \geq u^{-1}(n), s_{i}=\left(P_{u(i)} \mid Q_{v(i)}\right)$ and so there is a top-level occurrence of $a$ in each such $s_{i}$, contradicting $P \mid Q$ lock-free.

Suppose instead (2), that is, $p$ infinite but not fair. Then there exists a $P_{n}$ and an occurrence of an enabled action $a$ in $P_{n}$ s.t. no $\beta_{j}$ with $j \geq n$ executes that occurrence. By definition, every $P_{j} \stackrel{\beta_{j}}{\rightarrow} P_{j+1}$ then preserves that occurrence. By Lemma 6.1 (1) the occurrence in $P_{n}$ is top-level, and so by Lemma 6.1(2) it also is in every $P_{j}$. But for $j \geq u^{-1}(n), s_{j}=\left(P_{u(j)} \mid Q_{v(j)}\right)$, and so we have found a top-level occurrence of $a$ in each such $s_{j}$, contradicting $P \mid Q$ lock-free.

6.3. Primitive recursion. We proceed to establish our main result in steps, starting with simple processes. These arise as the body of primitive recursion.

Definition 6.11. A process $P$ is simple for $X$ iff

(1) no process variable but $X$ occurs free in $P$, and

(2) $\mathbf{0}$ is not a sub-term of $P$, and

(3) neither $\operatorname{rec} Y . Q$ nor $\left(\operatorname{rec}^{e} Y(i) . Q ; R\right)$ is a sub-term of $P$,

(4) $Q \mid R$ is not a sub-term of $P$.

Observe that by convention, in $\left(\operatorname{rec}^{e} X(i) . P ; Q\right), P$ is simple for $X$.

Lemma 6.12. If $P$ simple for $X$ and $s=\left(P_{i}, \lambda_{i}\right)_{i}$ is a maximal lock-free transition sequence of $P\{Q / X\}$, then $Q \stackrel{\lambda_{j-1}}{\longrightarrow} P_{j}$ for some $j>1$. 
Proof. By induction on $P$.

Case "0". Impossible: not simple for $X$.

Case " $k$ ! $\langle e\rangle . P$ ". Clearly $\left(P_{i+1}, \lambda_{i+1}\right)_{i}$ is a maximal lock-free transition sequence of $P\{\tilde{v} / \tilde{x}\}$. By the induction hypothesis $Q \stackrel{\lambda_{j}}{\longrightarrow} P_{j}$ for some $j>2$.

Case " $k ?(x) . P$ ". Clearly $s^{\prime}=\left(P_{i+1}, \lambda_{i+1}\right)_{i}$ is a maximal lock-free transition sequence of $P\{Q / X\}\{\tilde{v} / \tilde{x}\}$. Because $x$ bound, it is fresh for $Q$, so $P\{\tilde{v} / \tilde{x}\}\{Q / X\}=P\{Q / X\}\{\tilde{v} / \tilde{x}\}$ and $s^{\prime}$ is a maximal lock-free transition sequence of the latter. But then by the induction hypothesis $Q \stackrel{\lambda_{j}}{\longrightarrow} P_{j}$ for some $j>2$.

Case " $k ?\left\{l_{i} . P_{i}\right\}_{j \in J "}$ ". Like $k !\langle e\rangle . P$.

Case " $k ! l . P "$ ". Like $k !\langle e\rangle . P$

Case " $P \mid R$ ". Impossible: not simple for $X$.

Case "rec $X . P$ ". Impossible: not simple for $X$.

Case "( $\left.\operatorname{rec}^{e} Y(i) . P ; R\right)$ ". Impossible: not simple for $X$.

Case " $Y[\tilde{k}]$ ". By $P$ simple for $X$ we must have $X=Y$ whence $s$ is a transition sequence of $X\{Q / X\}=Q$; clearly $Q \stackrel{\lambda_{1}}{\rightarrow} P_{2}$.

Case "if $e$ then $P$ else $R$ ". Like $k ?\left\{l_{i} . P_{i}\right\}_{j \in J}$.

Lemma 6.13. If $s=\left(P_{i}, \lambda_{i}\right)_{i}$ is a maximal lock-free transition sequence of $\left(\operatorname{rec}^{e} X(i) . P ; Q\right)$ then $Q \stackrel{\lambda_{j-1}}{\longrightarrow} P_{j}$ for some $j>1$.

Proof. By induction on $n$. If $n=0$ then $s$ is a transition sequence of $Q$ iff it is of $\left(\operatorname{rec}^{0} X(i) . P ; Q\right)$, so clearly $Q \stackrel{\lambda_{1}}{\longrightarrow} P_{2}$. If instead $n=m+1$ observe that

$$
\left(\operatorname{rec}^{m+1} X(i) . P ; Q\right) \stackrel{\lambda_{1}}{\rightarrow} R \quad \text { iff } \quad P\{m / i\}\left\{\left(\operatorname{rec}^{m} X(i) . P ; Q\right) / X\right\} \stackrel{\lambda_{1}}{\rightarrow} R .
$$

Take $s^{\prime}$ to be the same as $s$ except $P_{1}=P\{m / i\}\left\{\left(\operatorname{rec}^{m} X(i) . P ; Q\right) / X\right\}$. Note that $s^{\prime}$ is maximal and lock-free. By convention $P$ and so $P\{m / i\}$ is simple for $X$. Then by Lemma 6.13 for some $j$ we have $Q \stackrel{\lambda_{j-1}}{\longrightarrow} P_{j}$.

6.4. Liveness. In this sub-section, we finally establish that well-typed, lock-free processes are indeed live. To this end, we will prove that the following syntactic function $\mathcal{A}(P)$ is in fact an under-approximation of the set of labels process $P$ must necessarily select when run. 
Definition 6.14. When $P$ is a process, we define $\mathcal{A}(P)$ inductively as follows.

$$
\begin{aligned}
\mathcal{A}(\mathbf{0}) & =\emptyset \\
\mathcal{A}(k !\langle e\rangle \cdot P) & =\mathcal{A}(P) \\
\mathcal{A}(k ?(x) \cdot P) & =\mathcal{A}(P) \\
\mathcal{A}\left(k ?\left\{l_{i} \cdot P_{i}\right\}_{i \in I}\right) & =\bigcap_{i \in I}\left(\left\{l_{i}\right\} \cup \mathcal{A}\left(P_{i}\right)\right) \\
\mathcal{A}(k ! l . P) & =\{l\} \cup \mathcal{A}(P) \\
\mathcal{A}(P \mid Q) & =\mathcal{A}(P) \cup \mathcal{A}(Q) \\
\mathcal{A}(\operatorname{rec} X \cdot P) & =\mathcal{A}(P) \\
\mathcal{A}\left(\operatorname{rec}^{e} X(i) \cdot P ; Q\right) & =\mathcal{A}(Q) \\
\mathcal{A}(X[\tilde{k}]) & =\emptyset \\
\mathcal{A}(\text { if e then } P \text { else } Q) & =\mathcal{A}(P) \cap \mathcal{A}(Q)
\end{aligned}
$$

Proposition 6.15. If $s=\left(P_{i}, \alpha_{i}\right)_{i}$ is a maximal lock-free transition sequence of $P\{Q / X\}$ $\mathcal{A}(P) \subseteq \operatorname{sel}(\alpha)$.

Proof. First, notation: if $\alpha$ is a sequence $\alpha_{1} \alpha_{2} \cdots$ we define $\operatorname{shift}(\alpha)=\alpha_{2} \cdots$. We proceed by induction on $P$.

Case "0". Immediate from $\mathcal{A}(\mathbf{0})=\emptyset$.

Case " $k$ ! $\langle e\rangle . P$ ". Clearly $\left(P_{i+1}, \alpha_{i+1}\right)_{i}$ is a maximal lock-free transition sequence of $P\{\tilde{Q} / \tilde{X}\}$. By the induction hypothesis $\mathcal{A}(P) \subseteq \operatorname{sel}(\operatorname{shift}(\alpha))=\operatorname{sel}(\alpha)$.

Case " $k ?(x) . P$ ". Clearly, for some $v,\left(P_{i+1}, \alpha_{i+1}\right)_{i}$ is a maximal lock-free transition sequence of $P\{\tilde{Q} / \tilde{X}\}\{v / x\}$. As $x$ is bound $P\{\tilde{Q} / \tilde{X}\}\{v / x\}=P\{v / x\}\{\tilde{Q} / \tilde{X}\}$. Using the induction hypothesis $\mathcal{A}(P)=\mathcal{A}(P\{v / x\}) \subseteq \operatorname{sel}(\operatorname{shift}(\alpha))=\operatorname{res}(() \alpha)$.

Case " $k ?\left\{l_{i} \cdot P_{i}\right\}_{i \in I}$ ". Like $k !\langle e\rangle . P$.

Case " $k ! l . P$ ". Like $k !\langle e\rangle . P$.

Case " $P \mid R$ ". By Lemma 6.10 there exists traces maximal lock-free transition sequences $p, q$ of $P\{\tilde{Q} / \tilde{X}\}, R\{\tilde{Q} / \tilde{X}\}$ with traces $\beta, \delta$ s.t. $\operatorname{sel}(\beta) \cup \operatorname{sel}(\delta)=\operatorname{sel}(\alpha)$. Using the induction hypothesis we find $\mathcal{A}(P) \cup \mathcal{A}(R) \subseteq \operatorname{sel}(\beta) \cup \operatorname{sel}(\delta)=\operatorname{sel}(\alpha)$.

Case "rec $Y . P$ ". $s$ is lock-free maximal transition sequence of $\operatorname{rec} Y . P\{\tilde{Q} / \tilde{X}\}$. Then taking $s^{\prime}$ to be the same as $s$ except $P_{1}=P\{\tilde{Q} / \tilde{X}\}\{\operatorname{rec} Y .(P\{\tilde{Q} / \tilde{X}\}) / Y\}$ we have a maximal lock-free transition sequence of the latter, also with trace $\alpha$. Using the induction hypothesis $\mathcal{A}(\operatorname{rec} X . P)=\mathcal{A}(P) \subseteq \operatorname{sel}(\alpha)$.

Case "rec ${ }^{e} Y(i) . P ; R$ ". $s$ is a lock-free maximal transition sequence of $\operatorname{rec}^{e} Y(I) . P ; R\{\tilde{Q} / \tilde{X}\}$. By Lemma 6.13 for some $j>1$ we have $R \stackrel{\lambda_{j-1}}{\longrightarrow} P_{j}$, and so

$$
R \stackrel{\lambda_{j-1}}{\longrightarrow} P_{j} \stackrel{\lambda_{j}}{\longrightarrow} P_{j+1} \cdots
$$

is a lock-free maximal transition sequence of $R$. The induction hypothesis now implies that $\mathcal{A}\left(\operatorname{rec}^{e} Y(i) . P ; R\right)=\mathcal{A}(R) \subseteq \operatorname{sel}\left(\operatorname{shift}^{j-2}(\alpha)\right) \subseteq \operatorname{sel}(\alpha)$.

Case " $Y[\tilde{k}]$ ". Immediate from $\mathcal{A}(Y[\tilde{k}])=\emptyset$.

Case "if $e$ then $P$ else $R$ ". Like $k ?\left\{l_{i} . P_{i}\right\}_{j \in J}$. 
Now comes the key lemma: the under-approximation $\mathcal{A}(P)$ is in a sense an overapproximation of the pending-response environment of a well-typed process. The Lemma can be read like this: what the process is committed to do $(L)$, less what it has done so far $(M(\Gamma))$, it will do before iterating $(\mathcal{A}(P))$.

Lemma 6.16. Suppose that $\Gamma ; L \vdash P \triangleright \Delta$. Define mappings $M((A, I, \Delta))=A$ and $M((L, \Delta))=L$, and

$$
M(\Gamma)=\bigcup_{X \in \operatorname{dom}(\Gamma)} M(\Gamma(X))
$$

Then $L \backslash M(\Gamma) \subseteq \mathcal{A}(P)$.

Proof. By induction on the derivation of $\Gamma ; L \vdash P \triangleright \Delta$.

Case [G-Inact]. By typing, $L=\emptyset$.

Case G-Out]. $\mathcal{A}(k !\langle e\rangle \cdot P)=\mathcal{A}(P) \supseteq L \backslash M(\Gamma)$, the latter by typing and the induction hypothesis.

Case [G.IN]. Ditto.

Case [GRA]. By the induction hypothesis for $i \in I$

$$
\left.\left(L \backslash\left\{l_{i}\right\}\right) \cup L_{i}\right) \backslash\left(M\left(\Gamma+l_{i}\right) \subseteq \mathcal{A}\left(P_{i}\right) .\right.
$$

Observe that $M\left(\Gamma+l_{i}\right)=M(\Gamma) \cup\left\{l_{i}\right\}$. We compute:

$$
\begin{aligned}
L \backslash M(\Gamma) & =\cap_{i \in I}(L \backslash M(\Gamma)) \\
& \subseteq \cap_{i \in I}\left(\left\{l_{i}\right\} \cup(L \backslash(M(\Gamma)))\right) \\
& =\cap_{i \in I}\left(\left\{l_{i}\right\} \cup\left(L \backslash\left(M(\Gamma) \cup l_{i}\right)\right)\right) \\
& =\cap_{i \in I}\left(\left\{l_{i}\right\} \cup\left(\left(L \backslash\left\{l_{i}\right\}\right) \backslash\left(M\left(\Gamma+l_{i}\right)\right)\right)\right) \\
& \subseteq \cap_{i \in I}\left(\left\{l_{i}\right\} \cup\left(\left(L \backslash\left\{l_{i}\right\}\right) \cup L_{i}\right) \backslash\left(M\left(\Gamma+l_{i}\right)\right)\right) \\
& \subseteq \cap_{i \in I}\left(\left\{l_{i}\right\} \cup \mathcal{A}\left(P_{i}\right)\right) \\
& =\mathcal{A}\left(k ?\left\{l_{i} . P_{i}\right\}_{i \in I}\right)
\end{aligned}
$$

Case [G.SEL]. Similar to [G-BRA].

Case [G-PAR]. By typing, we find $\Delta_{1}, \Delta_{2}$ and $L_{1}, L_{2}$ s.t. $\Gamma ; L_{i} \vdash P_{i} \triangleright \Delta_{i}$. By the induction hypothesis we then find that $L_{i} \backslash M(\Gamma) \subseteq \mathcal{A}\left(P_{i}\right)$. We now compute:

$$
\begin{aligned}
L \backslash M(\Gamma) & =\left(L_{1} \cup L_{2}\right) \backslash M(\Gamma) \\
& =L_{1} \backslash M(\Gamma) \cup L_{2} \backslash M(\Gamma) \\
& \subseteq \mathcal{A}\left(P_{1}\right) \cup \mathcal{A}\left(P_{2}\right) \\
& =\mathcal{A}\left(P_{1} \mid P_{2}\right)
\end{aligned}
$$

Case [G VARP]. We have $\Gamma, X:\left(L^{\prime}, \Delta\right) ; L \vdash X[\tilde{k}] \triangleright \Delta$. By typing $L \subseteq L^{\prime}$; by definition $L^{\prime} \subseteq M(\Gamma)$. But then $L \backslash M(\Gamma)=\emptyset$.

Case [G-ReCP]. We have $\Gamma ; L \vdash \operatorname{rec}^{e} X(i) . P ; Q \triangleright \Delta$. By typing we have $\Gamma \vdash Q \triangleright \Delta$ and by definition $\mathcal{A}\left(\operatorname{rec}^{e} X(i) . P ; Q\right)=\mathcal{A}(Q) \supseteq L \backslash M(\Gamma)$, the latter by the induction hypothesis.

Case [G-VAR]. We have $\Gamma, X:(A, I, \Delta) ; L \vdash X \triangleright \Delta$. By definition, we find $A \subseteq M(\Gamma)$, so by typing $L \subseteq I \subseteq A \subseteq M(\Gamma)$. But then $L \backslash M(\Gamma)=\emptyset$. 
Case G-REC]. We have $\Gamma ; L \vdash \operatorname{rec} X . P \triangleright \Delta$. By typing we must have $\Gamma, X:(\emptyset, I, \Delta) ; I \vdash$ $P \triangleright \Delta$. We compute.

$$
\begin{aligned}
L \backslash M(\Gamma) & \subseteq I \backslash(M(\Gamma) \cup \emptyset) \\
& =I \backslash M(\Gamma, X:(\emptyset, I, \Delta)) \\
& \subseteq \mathcal{A}(P)
\end{aligned}
$$

by $\mathrm{IH}$

Case [G-Cond]. By typing and the induction hypothesis we have $L \backslash \Gamma(M) \subseteq \mathcal{A}(P)$ and $L \backslash \Gamma(M) \subseteq \mathcal{A}(Q)$. But then also $L \backslash \Gamma(M) \subseteq \mathcal{A}(P) \cap \mathcal{A}(Q)=\mathcal{A}($ if $e$ then $P$ else $Q)$.

And this is enough: We can now prove that well-typed lock-free processes in fact do select every response mentioned in the "pending response" part of their type environment.

Proposition 6.17. Suppose $\cdot ; L \vdash P \triangleright \Delta$ with $P$ lock-free, and let $s=\left(P_{i}, \alpha_{i}\right)_{i}$ be a maximal transition sequence of $P$. Then $L \subseteq \operatorname{sel}(\alpha)$.

Proof. Observe that necessarily $s$ lock-free. We compute:

$$
\begin{aligned}
L & \subseteq \mathcal{A}(P) & & \text { By Lemma 6.16 } \\
& \subseteq \operatorname{sel}(\alpha) & & \text { By Proposition } 6.15
\end{aligned}
$$

Example 6.18. We saw in Example 5.14 that the process $D$ of Example 2.1 is typable $\cdot ;\{\mathrm{SI}\} \vdash D \triangleright \cdots$. By Proposition 6.17 above, noting that $D$ is clearly lock-free, every maximal transition sequence of $D$ must eventually select $\mathrm{SI}$.

Applying the definition of "live process" we now have our desired Theorem:

Theorem 6.19. Suppose $\cdot ; \vdash P \triangleright \Delta$ with $P$ lock-free. Then $P$ is live for $\cdot, \Delta$.

Proof. Consider a maximal transition sequence $\left(P_{i}, \alpha_{i}\right)$ of $P$. By Definition 6.7 we must find a live type transition sequence $\left(\Delta_{i}, \delta_{i}\right)$ of $\Delta$ with $\left(\left(P_{i}, \Delta_{i}\right),\left(\alpha_{i}, \delta_{i}\right)\right)$ a typed transition sequence of $\cdot \vdash P \triangleright \Delta$.

By induction and Theorem 5.13 there exists a sequence $\left(\Delta_{i}, L_{i}, \delta_{i}\right)_{i}$ with $\cdot L_{i} \vdash P_{i} \triangleright \Delta_{i}$

and $\Delta_{i} \stackrel{\delta_{i}}{\rightarrow} \Delta_{i+1}$ and $\delta_{i} \simeq \alpha_{i}$, and moreover $L_{i+1}=L_{i} \backslash \operatorname{res}\left(\delta_{i}\right) \cup \operatorname{req}\left(\delta_{i}\right)$. Suppose $l \in \operatorname{req}\left(\delta_{n}\right)$. Then $l \in L_{n+1}$. Clearly $P_{n+1}$ also lock-free, so by Proposition 6.15, $l \in \operatorname{sel}\left(\operatorname{shift}^{n}(\alpha)\right)$. That means there exists $j>n$ with $l \in \operatorname{sel}\left(\alpha_{j}\right)$. But $\alpha_{j} \simeq \delta_{j}$ so $l \in \operatorname{res}\left(\delta_{j}\right)$.

Example 6.20. We saw in Example 5.14 that $P(D)$ is typable as $\cdot ; \emptyset P(D) \triangleright k: T_{P}, o^{+}$: $T_{D}, o^{-}: \overline{T_{D}}$. Noting $P(D)$ lock-free, by the above Theorem it is live, and so will uphold the liveness guarantee in $T_{P}$ : if $\mathrm{CO}$ is selected, then eventually also $\mathrm{SI}$ is selected. Or in the intuition of the example: If the buyer performs "Checkout", he is guaranteed to subsequently receive an invoice.

\section{Conclusion and Future Work}

We introduced a conservative generalization of binary session types to session types with responses, which allows to specify request-response liveness properties. We showed that session types with responses are strictly more expressive (wrt. the classes of behaviours they can express) than standard binary session types. We provided a typing system for a process calculus similar to a non-trivial subset of collaborative BPMN processes with 
possibly infinite loops and bounded iteration and proved that lock-free, well typed processes are live.

We have identified several interesting directions for future work:

- The present typing system requires guessing suitable invariants $I$ for typing checking recursion, i.e., rec X.P. We believe that the syntactic approximation $\mathcal{A}(P)$ of Definition 6.14 is the unique maximal $I$ that will allow typing ${ }^{1}$. Lifting this belief to a Theorem would be an essential foundational step necessary for operationalising the present typing systems into a type-inference algorithm.

- The present techniques should be augmented by or combined with existing type systems for ensuring lock-freedom of session-typed processes (e.g., [16, 4, 8, 14, 29, 30]).

- The present work could be lifted to multi-party session types, which guarantees lockfreedom.

- The notion of request-response structure invites even more general notion of liveness, e.g., rather than requiring a particular future response, one might require at least one of a set of possible future responses.

- Channel passing is presently omitted for simplicity of presentation and not needed for our expressiveness result (Theorem 3.10). Introducing it, raises the question of whether one can delegate the responsibility for doing responses or not? If not, then channel passing does not affect the liveness properties of a lock-free process, and so is not really interesting for the present paper. If one could, it must be ensured that responses are not forever delegated without ever being fulfilled, which is an interesting challenge for future work. We hope to leverage existing techniques for the $\pi$-calculus, e.g., [24].

- Finally, and more speculatively, we plan to investigate relations to fair subtyping [28] and Live Sequence Charts [9].

\section{ACKNOWLEDGEMENT}

We gratefully acknowledge helpful comments from anonymous reviews at LMCS and various conferences.

Appendix A. SubJeCt REDUCTION PRoof For the STANDARD SESSION TYPING System

Lemma A.1 (Process variable substitution). Suppose that $\Theta, X: \Delta^{\prime} \vdash_{\text {std }} P \triangleright \Delta$. Suppose moreover that $\Theta \vdash_{\text {std }} Q \triangleright \Delta^{\prime}$ with $X$ is not free in $Q$. Then also $\Theta \vdash_{\text {std }} P\{Q / X\} \triangleright \Delta$

Proof. By induction on the typing derivation.

Case [G-Inact]. We have $\Theta, X: \Delta^{\prime} \vdash_{\text {std }} \mathbf{0} \triangleright \Delta$. By typing $\Delta$ completed, so by [G-InACT], we have $\Theta \vdash_{\text {std }} \mathbf{0}\{Q / X\} \triangleright \Delta$.

Case [G-OUT]. Immediate from the induction hypothesis.

Case [G-In]. Immediate from the induction hypothesis.

Case [G-BRA]. We have

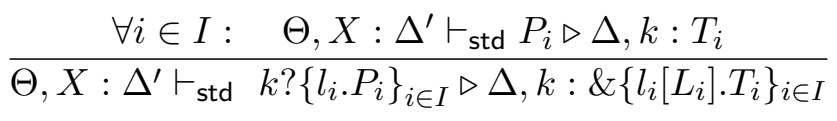

\footnotetext{
${ }^{1}$ We extend our gratitude to Anonymous Referee \#1 for pointing us in this direction.
} 
By the induction hypothesis and [G-BRA] we have

$$
\frac{\Theta \vdash_{\text {std }} P_{i}\{Q / X\} \triangleright \Delta, k: T_{i}}{\Theta \vdash_{\text {std }} k ?\left\{l_{i} \cdot P_{i}\{Q / X\}\right\}_{i \in I} \triangleright \Delta, k: \&\left\{l_{i}\left[L_{i}\right] \cdot T_{i}\right\}_{i \in I}} .
$$

Case [G-SEL]. Similar to [G-BRA].

Case [G-PAR]. We have $\Theta, X: \Delta^{\prime} \vdash_{\text {std }} P_{1} \mid P_{2} \triangleright \Delta$. By typing we find some $\Delta_{1}, \Delta_{2}=\Delta$ such that $\Theta, X: \Delta \vdash_{\text {std }} P_{i} \triangleright \Delta_{i}$. By induction we find $\Theta \vdash_{\text {std }} P_{i}\{Q / X\} \triangleright \Delta_{i}$, which in turn yields $\Theta \vdash_{\text {std }}\left(P_{1} \mid P_{2}\right)\{Q / X\} \triangleright \Delta_{1}, \Delta_{2}$.

Case [G-VARP]. Suppose first $X \neq Y$; then we have

so by [G.VARP] also

$$
\frac{\operatorname{dom}(\Delta)=\tilde{k}}{\Theta, Y: \Delta, X: \Delta^{\prime} \vdash_{\text {std }} Y[\tilde{k}] \triangleright \Delta},
$$

$$
\Theta, Y: \Delta \vdash_{\text {std }} Y[\tilde{k}]\{Q / X\} \triangleright \Delta .
$$

If on the other hand $X=Y$ we have by typing

$$
\frac{\operatorname{dom}(\Delta)=\tilde{k}}{\Theta, X: \Delta^{\prime} \vdash_{\text {std }} X[\tilde{k}] \triangleright \Delta} ;
$$

and it must be the case that $\Delta=\Delta^{\prime}$. We have by assumption $\Theta \vdash_{\text {std }} Q \triangleright \Delta^{\prime}$, that is $\Theta \vdash_{\text {std }} X[\tilde{k}]\{Q / X\} \triangleright \Delta$.

Case [G-RecP]. We have $\Theta, X: \Delta^{\prime} \vdash_{\text {std }}\left(\operatorname{rec}^{e} Y(i) . P ; R\right) \triangleright \Delta$. By typing we have $\Theta, X$ : $\Delta^{\prime}, Y: \Delta \vdash_{\text {std }} P \triangleright \Delta$ and $\Theta, X: \Delta^{\prime} \vdash_{\text {std }} R \triangleright \Delta$. Using $\Theta \vdash_{\text {std }} Q \triangleright \Delta^{\prime}$, by induction $\Theta, Y: \Delta \vdash_{\text {std }}$ $P\{Q / X\} \triangleright \Delta$ and $\Theta \vdash_{\text {std }} R\{Q / X\} \triangleright \Delta$, which in turn yields $\Theta \vdash_{\text {std }^{\prime}}\left(\operatorname{rec}^{e} Y(i) . P ; R\right)\{Q / X\} \triangleright \Delta$. Case [G.VAR]. Suppose first $X \neq Y$; then by typing we have

$$
\frac{\operatorname{dom}(\Delta)=\tilde{k}}{\Theta, Y: \Delta, X: \Delta^{\prime} \vdash_{\text {std }} Y[\tilde{k}] \triangleright \Delta},
$$

so by [G-VAR] also

$$
\Theta, Y: \Delta \vdash_{\text {std }} Y[\tilde{k}]\{Q / X\} \triangleright \Delta
$$

If on the other hand $X=Y$ we have by typing

$$
\frac{\operatorname{dom}(\Delta)=\tilde{k}}{\Theta, X: \Delta^{\prime} \vdash_{\text {std }} X[\tilde{k}] \triangleright \Delta},
$$

where necessarily $\Delta=\Delta^{\prime}$, so $\Theta \vdash_{\text {std }} X[\tilde{k}]\{Q / X\} \triangleright \Delta^{\prime}$.

Case [GREC]. We have $\Theta, X: \Delta^{\prime} \vdash_{\text {std }} \operatorname{rec} Y . P \triangleright \Delta$. We find by typing $\Theta, X: \Delta^{\prime}, Y: \Delta \vdash_{\text {std }}$ $P \triangleright \Delta$, hence by the induction hypothesis $\Theta, Y: \Delta^{\prime} \vdash_{\text {std }} P\{Q / X\} \triangleright \Delta$, and so by [GR REC] $\Theta \vdash_{\text {std }}(\operatorname{rec} Y . P)\{Q / X\} \triangleright \Delta$.

Case [G-COND]. Immediate from the induction hypothesis.

Lemma A.2. If $\Theta \vdash_{\text {std }} P \triangleright \Delta$ then also $\Theta \vdash_{\text {std }} P\{v / x\} \triangleright \Delta$.

Proof. Straightforward induction.

Lemma A.3. If $\Theta \vdash_{\text {std }} P \triangleright \Delta$ and $\Delta(k) \neq$ end then $k \in \mathrm{fn}(P)$.

Proof. Straightforward induction. 
Lemma A.4. Suppose $\Theta \vdash_{\text {std }} P \triangleright \Delta, k: T$ with $\Delta, k: T$ balanced, $T \neq$ end, and $\bar{k} \notin \mathrm{fn}(P)$. Then $\bar{k} \notin \operatorname{dom}(\Delta)$.

Proof. Supposed for a contradiction $\bar{k} \in \operatorname{dom}(\Delta)$. Because $\Delta, k: ! . T$ balanced, $\Delta(\bar{k}) \neq$ end. By Lemma A.3 we thus have $\bar{k} \in \mathrm{fn}(P)$; contradiction.

Lemma A.5. Suppose that $\Theta \vdash_{\text {std }} P \triangleright \Delta$ with $P \stackrel{\lambda}{\rightarrow} Q$. Then there exists a type transition $\Delta \stackrel{\delta}{\rightarrow} \Delta^{\prime}$ with $\delta \simeq \lambda$, such that $\Theta \vdash_{\text {std }} Q \triangleright \Delta^{\prime}$. Moreover, if $\Delta$ balanced, then also $\Delta^{\prime}$ balanced.

Proof. By induction on the derivation of the transition.

Case [C-Out]. We have $k !\langle e\rangle . P \stackrel{k ! v}{\longrightarrow} P$ with $\bar{k} \notin P$ and $\Theta \vdash_{\text {std }} k !\langle e\rangle . P \triangleright \Delta, k: ! . T$. By typing $\Theta \vdash_{\text {std }} P \triangleright \Delta, k: T$. By [F-LIFT] we have $k: ! . T \stackrel{k: !}{\longrightarrow} k: T$. By [F-PAR] $\Delta, k: ! . T \stackrel{k: !}{\longrightarrow} \Delta, k: T$. Observing that $k: ! \simeq k ! v$ we have found the requisite type transition.

Now suppose $\Delta, k: ! . T$ balanced; we must show $\Delta, k: T$ balanced. It is sufficient to show $\bar{k} \notin \operatorname{dom}(\Delta)$. But this follows from Lemma A.4.

Case [C-In]. We have $k ?(x) . P \stackrel{k ? v}{\longrightarrow} P\{v / x\}$ with $\bar{k} \notin \mathrm{fn}(P)$ and $\Theta \vdash_{\text {std }} k ?(x) . P \triangleright \Delta, k:$ ?.T. By typing $\Theta \vdash_{\text {std }} P \triangleright \Delta, k: T$. By [F-LifT] and [F-PAR], $\Delta, k: ? . T \stackrel{k: ?}{\longrightarrow} \Delta, k: T$. By Lemma A.2 we have $\Theta \vdash_{\text {std }} P\{v / x\} \triangleright \Delta, k: T$. Observing $k: ? \simeq k ? v$ we have found the requisite transition and typing. Preservation of balance follows from Lemma A.4.

Case [C-BRA]. We have $k ?\left\{l_{i} . P_{i}\right\} \stackrel{k \& l_{i}}{\longrightarrow} P_{i}$ and $\Theta \vdash_{\text {std }} k ?\left\{l_{i} . P_{i}\right\}_{i \in I} \triangleright \Delta, k: \&\left\{l_{i}\left[L_{i}\right] . T_{i}\right\}_{i \in I}$. By typing we have $\Theta+l_{i} \vdash_{\text {std }} P_{i} \triangleright \Delta, k: T_{i}$. By [F-LifT] and [F-PAR] we have $\Delta, k:$ $\&\left\{l_{i}\left[L_{i}\right] . T_{i}\right\}_{i \in I} \stackrel{k: \& l_{i}\left[L_{i}\right]}{\longrightarrow} \Delta, k: T_{i}$. Observing that $k: \oplus l_{i}\left[L_{i}\right] \simeq k \& l_{i}$, we have found the requisite type transition. Preservation of balance follows from Lemma A.4.

Case [C-SEL]. We have $k ! l . P \stackrel{k \oplus l_{i}}{\longrightarrow} P$ and $\Theta \vdash_{\text {std }} k ! l_{i} . P \triangleright \Delta, k: \oplus\left\{l_{i}\left[L_{i}\right] . T_{i}\right\}_{i \in I}$. By typing $\Theta+l_{i} \vdash_{\text {std }} P \triangleright \Delta, k: T_{i}$. By [F-LifT] and [F-PAR] we have

$$
\Delta, \oplus\left\{l_{i}\left[L_{i}\right] . T_{i}\right\}_{i \in I} \stackrel{k: \oplus l_{i}\left[L_{i}\right]}{\longrightarrow} \Delta, T_{i} .
$$

Observing that $k: \oplus l_{i}\left[L_{i}\right] \simeq k \oplus l_{i}$, we have found the requisite type transition. Preservation of balance follows from Lemma A.4.

Case [C-PARL]. We have $P\left|P^{\prime} \stackrel{\lambda}{\rightarrow} Q\right| P^{\prime}$ with $\overline{\operatorname{subj}(\lambda)} \notin \mathrm{fn}\left(P^{\prime}\right)$ and $\Theta \vdash_{\text {std }} P \mid P^{\prime} \triangleright \Delta$. By typing we have for some $L_{1} \cup L_{2}=L$ and $\Delta_{1} \cup \Delta_{2}$ that $\Theta_{1} \vdash_{\text {std }} P \triangleright \Delta_{1}$ and $\Theta \vdash_{\text {std }} P^{\prime} \triangleright \Delta_{2}$. By the induction hypothesis, we have a transition $\Delta_{1} \stackrel{\delta}{\rightarrow} \Delta_{1}^{\prime}$ with $\Theta \vdash_{\text {std }} Q \triangleright \Delta_{1}^{\prime}$ and $\delta \simeq \lambda$. By Lemma $4.5 \operatorname{dom}\left(\Delta_{1}\right)=\operatorname{dom}\left(\Delta_{1}^{\prime}\right)$ so $\Delta_{1}^{\prime}, \Delta_{2}$ is defined, and hence by [G.PAR] we have $\Theta \vdash_{\text {std }} Q \mid P^{\prime} \triangleright \Delta_{1}^{\prime}, \Delta_{2}$ and thus the requisite transition.

Now suppose $\Delta_{1}, \Delta_{2}$ balanced. By Lemma 4.7 it is sufficient to prove that $\overline{\operatorname{subj}(\delta)} \notin$ $\operatorname{dom}\left(\Delta_{1}, \Delta_{2}\right)$. If $\operatorname{subj}(\delta)=\tau$ this is trivial, so say $\operatorname{subj}(\delta)=k$ and suppose for a contradiction $\bar{k} \in \operatorname{dom}\left(\Delta_{1}, \Delta_{2}\right)$. We must have $\delta=k: \rho$ and because $\delta \simeq \lambda$ we must have $\operatorname{subj}(\lambda)=$ $\operatorname{subj}(\delta)=k$. By Lemma $2.2 \bar{k} \notin \mathrm{fn}\left(Q \mid P^{\prime}\right)$. By Lemma 4.6 we have $\Delta_{1}=\Delta_{1}^{\prime \prime}, k: S$ with $S \neq$ end. Because $\Delta_{1}, \Delta_{2}$ balanced, $\left(\Delta_{1}, \Delta_{2}\right)(\bar{k}) \bowtie S$ and so $\left(\Delta_{1}, \Delta_{2}\right)(\bar{k}) \neq$ end.

Suppose first $\bar{k} \in \operatorname{dom}\left(\Delta_{1}\right)$. Then $\bar{k} \in \operatorname{dom}\left(\Delta_{1}^{\prime \prime}\right)$, so also $\Delta^{\prime \prime}(\bar{k}) \neq$ end, and it follows that $\Delta_{1}^{\prime}(\bar{k})=\Delta_{1}^{\prime \prime}(\bar{k}) \neq$ end. By Lemma $5.10 \bar{k} \in \mathrm{fn}(Q)$, contradicting $\bar{k} \notin \mathrm{fn}\left(Q \mid P^{\prime}\right)$.

Suppose instead $\bar{k} \in \operatorname{dom}\left(\Delta_{2}\right)$. Then immediately by Lemma $5.10 \bar{k} \in \mathrm{fn}\left(P^{\prime}\right)$, contradicting $\bar{k} \notin \mathrm{fn}\left(Q \mid P^{\prime}\right)$. 
Case $[\mathrm{COM}-1]$. We have

$$
\frac{P_{1} \stackrel{\bar{k} ! v}{\longrightarrow} P_{1}^{\prime} \quad P_{2} \stackrel{k ? v}{\rightarrow} P_{2}^{\prime}}{P_{1}\left|P_{2} \stackrel{\tau}{\rightarrow} P_{1}^{\prime}\right| P_{2}^{\prime}}
$$

and

$$
\frac{\Theta \vdash_{\text {std }} P_{1} \triangleright \Delta_{1} \quad \Theta \vdash_{\text {std }} P_{2} \triangleright \Delta_{2}}{\Theta \vdash_{\text {std }} P_{1} \mid P_{2} \triangleright \Delta_{1}, \Delta_{2}}
$$

By induction we find $\Delta_{i} \stackrel{\delta_{i}}{\rightarrow} \Delta_{i}^{\prime}$ s.t. $\Theta \vdash_{\text {std }} P_{I} \triangleright \Delta_{i}^{\prime}$ with $\delta_{1} \simeq \bar{k} ! v$ and $\delta_{2} \simeq k ? v$. It follows that $\delta_{1}=k:$ ! and $\delta_{2}=k:$ ?. By [F-Com1] we thus have $\Delta_{1}, \Delta_{2} \stackrel{\tau}{\rightarrow} \Delta_{1}^{\prime}, \Delta_{2}^{\prime}$. Noting that $\tau \simeq \tau$, we have the required type transition. $\operatorname{Since} \operatorname{subj}(\tau)=\tau$ and $\operatorname{so} \overline{\operatorname{subj}(\tau)} \notin \operatorname{dom}\left(\Delta_{1}, \Delta_{2}\right)$, it follows from Lemma 4.7 that $\Delta_{1}^{\prime}, \Delta_{2}^{\prime}$ is balanced when $\Delta_{1}, \Delta_{2}$ is.

Case [C-Com2]. We have

and

$$
\frac{P_{1} \stackrel{\bar{k} \oplus l}{\longrightarrow} P_{1}^{\prime} \quad P_{2} \stackrel{k \& l}{\longrightarrow} P_{2}^{\prime}}{P_{1}\left|P_{2} \stackrel{\tau: l}{\longrightarrow} P_{1}^{\prime}\right| P_{2}^{\prime}}
$$

$$
\frac{\Theta \vdash_{\text {std }} P_{1} \triangleright \Delta_{1} \quad \Theta \vdash_{\text {std }} P_{2} \triangleright \Delta_{2}}{\Theta \vdash_{\text {std }} P_{1} \mid P_{2} \triangleright \Delta_{1}, \Delta_{2}}
$$

By induction we find $\Delta_{i} \stackrel{\delta_{i}}{\rightarrow} \Delta_{i}^{\prime}$ s.t. $\Theta \vdash_{\text {std }} P_{i} \triangleright \Delta_{i}^{\prime}$ with $\delta_{1} \simeq \bar{k} \oplus l$ and $\delta_{2} \simeq k \& l$. It follows that $\delta_{1}=\bar{k}: \oplus l\left[L_{1}^{\prime}\right]$, and so we find a transition $\Delta_{1}, \Delta_{2} \stackrel{\tau: l, L_{1}^{\prime} \cup L_{2}^{\prime}}{\longrightarrow} \Delta_{1}^{\prime}, \Delta_{2}^{\prime}$ by [F-Com2]. By G.PAR] we find $\Theta \vdash_{\text {std }} P_{1}^{\prime} \mid P_{2}^{\prime} \triangleright \Delta_{1}^{\prime}, \Delta_{2}^{\prime}$. Noting that $\tau: l, L_{1}^{\prime} \cup L_{2}^{\prime} \simeq \tau: l$ we have the required type transition. Since $\operatorname{subj}\left(\tau: l, L_{1}^{\prime} \cup L_{2}^{\prime}\right)=\tau$ and $\operatorname{so} \overline{\operatorname{subj}\left(\tau: l, L_{1}^{\prime} \cup L_{2}^{\prime}\right)} \notin \operatorname{dom}\left(\Delta_{1}, \Delta_{2}\right)$, it follows from Lemma 4.7 that $\Delta_{1}^{\prime}, \Delta_{2}^{\prime}$ is balanced when $\Delta_{1}, \Delta_{2}$ is.

Case [C-REC]. We have

and

$$
\frac{P\{\operatorname{rec} X . P / X\} \stackrel{\lambda}{\rightarrow} Q}{\operatorname{rec} X . P \stackrel{\lambda}{\rightarrow} Q}
$$

It then follows by Lemma A.1 that

$$
\frac{\Theta, X: \Delta \vdash_{\text {std }} P \triangleright \Delta}{\Theta \vdash_{\text {std }} \operatorname{rec} X . P \triangleright \Delta}
$$

$$
\Theta \vdash_{\text {std }} P\{\operatorname{rec} X . P / X\} \triangleright \Delta,
$$

and so by induction we find the required balance-preserving type transition.

Case [C-PREC0]. We have

and

$$
\frac{e \Downarrow 0 \quad Q \stackrel{\lambda}{\rightarrow} R}{\left(\operatorname{rec}^{e} X(i) . P ; Q\right) \stackrel{\lambda}{\rightarrow} R}
$$

$$
\frac{\Theta, X: \Delta \vdash_{\text {std }} P \triangleright \Delta \quad \Theta \vdash_{\text {std }} R \triangleright \Delta}{\Theta \vdash_{\text {std }}\left(\operatorname{rec}^{e} X(i) . P ; Q\right) \triangleright \Delta},
$$

and so by the induction hypothesis we find the required balance-preserving type transition. Case [C-PrecN]. We have

$$
\frac{e \Downarrow n+1 \quad P\{n / i\}\left\{\left(\operatorname{rec}^{n} X(i) \cdot P ; Q\right) / X\right\} \stackrel{\lambda}{\rightarrow} R}{\left(\operatorname{rec}^{e} X(i) \cdot P ; Q\right) \stackrel{\lambda}{\rightarrow} R}
$$


and again

$$
\frac{\Theta, X: \Delta \vdash_{\text {std }} P \triangleright \Delta \quad \Theta \vdash_{\text {std }} Q \triangleright \Delta}{\Theta \vdash_{\text {std }}\left(\operatorname{rec}^{e} X(i) . P ; Q\right) \triangleright \Delta} .
$$

By Lemma A.2 we have $\Theta, X:\left(L^{\prime}, \Delta\right) \vdash_{\text {std }} P\{n / i\} \triangleright \Delta$. By Lemma A.1 we have

$$
\Theta \vdash_{\text {std }} P\{n / i\}\left\{\left(\operatorname{rec}^{n} X(i) . P ; Q\right) / X\right\} \triangleright \Delta,
$$

and the requisite balance-preserving type transition follows by the induction hypothesis. Case [C-CondT] and [C-CondF]. We have

$$
\frac{e \Downarrow \text { true } \quad P \stackrel{\lambda}{\rightarrow} P^{\prime}}{\text { if } e \text { then } P \text { else } Q \stackrel{\lambda}{\rightarrow} P^{\prime}}
$$

and

$$
\frac{\Theta \vdash P \triangleright \Delta \quad \Theta \vdash Q \triangleright \Delta}{\Theta \vdash \text { if } e \text { then } P \text { else } Q \triangleright \Delta},
$$

and the requisite balance-preserving type transition follows from the induction hypothesis. 


\section{REFERENCES}

[1] Bettini, L., M. Coppo, L. D’Antoni, M. D. Luca, M. Dezani-Ciancaglini and N. Yoshida, Global progress in dynamically interleaved multiparty sessions, in: CONCUR, 2008, pp. 418-433.

[2] Brill, M., W. Damm, J. Klose, B. Westphal and H. Wittke, Live sequence charts: An introduction to lines, arrows, and strange boxes in the context of formal verification, in: SoftSpez Final Report, LNCS 3147 (2004), pp. 374-399.

[3] Cacciagrano, D., F. Corradini and C. Palamidessi, Explicit fairness in testing semantics, Logical Methods in Computer Science 5 (2009). URL http://arxiv .org/abs/0904.2340

[4] Caires, L. and F. Pfenning, Session types as intuitionistic linear propositions, in: P. Gastin and F. Laroussinie, editors, CONCUR 2010 - Concurrency Theory, Lecture Notes in Computer Science 6269, Springer Berlin Heidelberg, 2010 pp. 222-236. URL http://dx.doi.org/10.1007/978-3-642-15375-4_16

[5] Carbone, M. and S. Debois, A graphical approach to progress for structured communication in web services, in: ICE, 2010, pp. 13-27.

[6] Cheung, S.-C., D. Giannakopoulou and J. Kramer, Verification of liveness properties using compositional reachability analysis, in: ESEC/SIGSOFT FSE, Lecture Notes in Computer Science 1301 (1997), pp. 227-243.

[7] Coppo, M., M. Dezani-Ciancaglini, L. Padovani and N. Yoshida, Inference of global progress properties for dynamically interleaved multiparty sessions, in: COORDINATION, 2013, pp. 45-59.

[8] Coppo, M., M. Dezani-Ciancaglini and N. Yoshida, Asynchronous session types and progress for object oriented languages, in: M. Bonsangue and E. Johnsen, editors, Formal Methods for Open Object-Based Distributed Systems, Lecture Notes in Computer Science 4468, Springer Berlin Heidelberg, 2007 pp. $1-31$. URL http://dx.doi .org/10.1007/978-3-540-72952-5_1

[9] Damm, W. and D. Harel, Lscs: Breathing life into message sequence charts, Formal Methods in System Design 19 (2001), pp. 45-80.

[10] Dardha, O., E. Giachino and D. Sangiorgi, Session types revisited, in: PPDP, 2012, pp. 139-150.

[11] Debois, S., T. T. Hildebrandt, T. Slaats and N. Yoshida, Type checking liveness for collaborative processes with bounded and unbounded recursion, in: E. Ábrahám and C. Palamidessi, editors, Formal Techniques for Distributed Objects, Components, and Systems - 34th IFIP WG 6.1 International Conference, FORTE 2014, Held as Part of the 9th International Federated Conference on Distributed Computing Techniques, DisCoTec 2014, Berlin, Germany, June 3-5, 2014. Proceedings, Lecture Notes in Computer Science 8461 (2014), pp. 1-16.

URL http://dx.doi .org/10.1007/978-3-662-43613-4_1

[12] Deniélou, P.-M. and N. Yoshida, Multiparty session types meet communicating automata, in: ESOP, 2012, pp. 194-213.

[13] Deniélou, P.-M. and N. Yoshida, Multiparty compatibility in communicating automata: Characterisation and synthesis of global session types, in: ICALP, 2013, pp. 174-186.

[14] Dezani-Ciancaglini, M., U. de'Liguoro and N. Yoshida, On progress for structured communications, in: G. Barthe and C. Fournet, editors, Trustworthy Global Computing, Lecture Notes in Computer Science 4912, Springer Berlin Heidelberg, 2008 pp. 257-275.

URL http://dx .doi .org/10.1007/978-3-540-78663-4_18

[15] Dezani-Ciancaglini, M., S. Drossopoulou, D. Mostrous and N. Yoshida, Objects and session types, Inf. Comput. 207 (2009), pp. 595-641.

[16] Dezani-Ciancaglini, M., D. Mostrous, N. Yoshida and S. Drossopoulou, Session types for object-oriented languages, in: D. Thomas, editor, ECOOP 2006 - Object-Oriented Programming, Lecture Notes in Computer Science 4067, Springer Berlin Heidelberg, 2006 pp. 328-352. URL http://dx.doi.org/10.1007/11785477_20

[17] Fossati, L., K. Honda and N. Yoshida, Intensional and extensional characterisation of global progress in the $\pi$-calculus, in: CONCUR, 2012, pp. 287-301.

[18] Gay, S. and M. Hole, Subtyping for session types in the pi calculus, Acta Informatica 42 (2005), pp. 191-225.

[19] Gay, S. J. and M. Hole, Subtyping for session types in the pi calculus, Acta Inf. 42 (2005), pp. 191-225. 
[20] Honda, K., A. Mukhamedov, G. Brown, T.-C. Chen and N. Yoshida, Scribbling interactions with a formal foundation, in: ICDCIT, 2011, pp. 55-75.

[21] Honda, K., V. Vasconcelos and M. Kubo, Language primitives and type discipline for structured communication-based programming, in: ESOP, 1998, pp. 122-138.

[22] Honda, K., N. Yoshida and M. Carbone, Multiparty asynchronous session types, in: POPL, 2008, pp. $273-284$.

[23] Hu, R., N. Yoshida and K. Honda, Session-based distributed programming in Java, in: J. Vitek, editor, ECOOP '08, LNCS 5142, 2008 pp. 516-541.

[24] Kobayashi, N., A type system for lock-free processes, I\&C 177 (2002), pp. 122-159.

[25] Kobayashi, N. and C.-H. L. Ong, A type system equivalent to the modal mu-calculus model checking of higher-order recursion schemes, in: LICS (2009), pp. 179-188.

[26] Mostrous, D. and V. T. Vasconcelos, Session typing for a featherweight Erlang, in: COORDINATION, 2011, pp. 95-109.

[27] Object Management Group BPMN Technical Committee, Business Process Model and Notation, v2.0, Webpage (2011), http://www.omg.org/spec/BPMN/2.0/PDF

[28] Padovani, L., Fair subtyping for open session types, in: ICALP, 2013, pp. 373-384.

[29] Padovani, L., From lock freedom to progress using session types, in: PLACES 2013, EPTCS 137, 2013, pp. 3-19.

[30] Padovani, L., Deadlock and lock freedom in the linear $\pi$-calculus, in: Proceedings of the Joint Meeting of the Twenty-Third EACSL Annual Conference on Computer Science Logic (CSL) and the Twenty-Ninth Annual ACM/IEEE Symposium on Logic in Computer Science (LICS), CSL-LICS '14 (2014), pp. 72:1$72: 10$ URL http://doi.acm.org/10.1145/2603088.2603116

[31] Roa, J., O. Chiotti and P. D. Villarreal, A verification method for collaborative business processes, in: Business Process Management Workshops (1), Lecture Notes in Business Information Processing 99 (2011), pp. 293-305.

[32] Vasconcelos, V., Fundamentals of session types, I\&C 217 (2012), pp. 52-70.

[33] Vieira, H. T. and V. T. Vasconcelos, Typing progress in communication-centred systems, in: COORDINATION, 2013, pp. 236-250.

[34] Yoshida, N. and V. T. Vasconcelos, Language primitives and type discipline for structured communicationbased programming revisited: Two systems for higher-order session communication, ENTCS 171 (2007), pp. 73-93. 